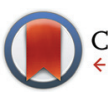

CrossMark \&lick for updates

Cite this: Dalton Trans., 2015, 44, 9569

Received 20th March 2015,

Accepted 20th April 2015

DOI: 10.1039/c5dt01120e

www.rsc.org/dalton

\title{
Hydrothermal synthesis of Group 13 metal trifluoride complexes with neutral $\mathrm{N}$-donor ligands $\dagger$
}

\author{
Rajiv Bhalla, ${ }^{a}$ William Levason, ${ }^{b}$ Sajinder K. Luthra, ${ }^{c}$ Graeme McRobbie, ${ }^{c}$ \\ Francesco M. Monzittu, ${ }^{\mathrm{b}}$ Jazmyn Palmer, ${ }^{\mathrm{b}}$ Gillian Reid, ${ }^{* \mathrm{~b}}$ George Sanderson ${ }^{\mathrm{b}}$ and \\ Wenjian Zhang ${ }^{\mathrm{b}}$
}

\begin{abstract}
The reactions of the hydrated Group 13 fluorides, $\mathrm{MF}_{3} \cdot 3 \mathrm{H}_{2} \mathrm{O}(\mathrm{M}=\mathrm{Al}$, Ga or In) with 2,2':6',2"'-terpyridyl, 2,2'-bipyridyl or 1,10-phenanthroline under hydrothermal conditions $\left(180{ }^{\circ} \mathrm{C} / 15 \mathrm{~h}\right)$ produced high yields of the complexes $\left[\mathrm{MF}_{3}(\right.$ terpy) $] \cdot 3 \mathrm{H}_{2} \mathrm{O},\left[\mathrm{MF}_{3}(\right.$ bipy $\left.)\left(\mathrm{OH}_{2}\right)\right] \cdot 2 \mathrm{H}_{2} \mathrm{O}$ and $\left[\mathrm{MF}_{3}\right.$ (phen) $\left.\left(\mathrm{OH}_{2}\right)\right]$. X-Ray crystal structures of $\left[M^{\prime} F_{3}(\right.$ terpy $\left.)\right] \cdot 3 \mathrm{H}_{2} \mathrm{O}\left(\mathrm{M}^{\prime}=\mathrm{Al}\right.$ or $\left.\mathrm{Ga}\right)$, $\left[\mathrm{M}^{\prime} \mathrm{F}_{3}(\right.$ bipy $\left.)\left(\mathrm{OH}_{2}\right)\right] \cdot 2 \mathrm{H}_{2} \mathrm{O}$ and $\left[\mathrm{GaF}_{3}(\right.$ phen $\left.)\left(\mathrm{OH}_{2}\right)\right]$ show that all of them contain distorted octahedral geometries at the metal with mer-trifluoride coordination. Extensive $\mathrm{H}$-bonding $(\mathrm{F} \cdots \mathrm{H}-\mathrm{OH})$ links the molecules. The complexes have been further characterised by microanalysis, IR, ${ }^{1} \mathrm{H},{ }^{19} \mathrm{~F}\left\{{ }^{1} \mathrm{H}\right\}$ and ${ }^{27} \mathrm{Al}$ NMR spectroscopy. In contrast, reactions of the trifluorides with the acyclic triamine, $N, N, N^{\prime}, N^{\prime}, N^{\prime \prime}$-pentamethyldiethylenetriamine, under similar hydrothermal conditions results in cleavage of the triamine and ring-closure to form the 1,1,4-trimethylpiperazinium cation, $\left[\subset \mathrm{Me}_{2} \mathrm{~N}\right.$ $\left.\left(\mathrm{CH}_{2}\right)_{2} \mathrm{NMe}\left(\mathrm{CH}_{2}\right)_{2}\right]^{+}$, with fluorometallate anions, and confirmed by $\mathrm{X}$-ray analysis of $\left[\subset \mathrm{CMe}_{2} \mathrm{~N}\left(\mathrm{CH}_{2}\right)_{2} \mathrm{NMe}\right.$ $\left.\left(\mathrm{CH}_{2}\right)_{2}\right]_{2}\left[\mathrm{Al}_{2} \mathrm{~F}_{8}\left(\mathrm{OH}_{2}\right)_{2}\right] \cdot 2 \mathrm{H}_{2} \mathrm{O}$. The strongly $\mathrm{H}$-bonded $\left[\mathrm{GaF}_{3}(\right.$ terpy) $] \cdot 3 \mathrm{H}_{2} \mathrm{O}$ was also obtained by $\mathrm{Cl} / \mathrm{F}$ exchange from $\left[\mathrm{GaCl}_{3}\left(\right.\right.$ terpy)] and $\left[\mathrm{NBu}_{4}\right] \mathrm{F}$ or $\left[\mathrm{K}\left(2,2,2\right.\right.$-crypt)]F. Crystallisation of a mixture of $\left[\mathrm{NH}_{4}\right]\left[\mathrm{PF}_{6}\right]$ and $\left[\mathrm{GaF}_{3}(\text { terpy) }] \cdot 3 \mathrm{H}_{2} \mathrm{O} \text { from aqueous solution produced the edge-bridged cationic complex, [\{Ga(terpy)F }\right\}_{2^{-}}$ $\left.(\mu-F)_{2}\right]\left[\mathrm{PF}_{6}\right]_{2}$. The synthesis of the more sterically bulky $\left[\mathrm{GaCl}_{3}\left({ }^{t} \mathrm{Bu}_{3}\right.\right.$-terpy)] $\left({ }^{\mathrm{t}} \mathrm{Bu} u_{3}\right.$-terpy $=4,4^{\prime} 4^{\prime \prime}-$ tris- ${ }^{t} \mathrm{Bu}-2,2^{\prime}: 6^{\prime}, 2^{\prime \prime}$-terpyridyl) and the crystal structure of $\left[\mathrm{GaCl}_{2}\left({ }^{\mathrm{t}} \mathrm{Bu}_{3}\right.\right.$-terpy)][$\left[\mathrm{GaCl}_{4}\right]$, which contains a trigonal bipyramidal cation, are also reported.
\end{abstract}

\section{Introduction}

The anhydrous Group 13 metal fluorides $\mathrm{MF}_{3}(\mathrm{M}=\mathrm{Al}, \mathrm{Ga}$ or In) are inert polymeric solids and generally unreactive towards neutral ligands. This has hindered attempts to explore their coordination chemistry, hence very few adducts have been described. ${ }^{1}$ The hydrates, $\mathrm{MF}_{3} \cdot 3 \mathrm{H}_{2} \mathrm{O}$, are more reactive, but have very poor solubility in organic solvents or water. ${ }^{1}$ Two structural forms of $\mathrm{AlF}_{3} \cdot 3 \mathrm{H}_{2} \mathrm{O}$ are known, the $\alpha$-form with discrete $\left[\mathrm{AlF}_{3}\left(\mathrm{OH}_{2}\right)_{3}\right]$ molecules, and the $\beta$-form which is a polymer, $\left[\left\{\mathrm{AlF}_{2}\left(\mathrm{OH}_{2}\right)_{2}(\mu-\mathrm{F})\right\}_{n}\right] \cdot n \mathrm{H}_{2} \mathrm{O} .^{2}$ The structure of $\mathrm{GaF}_{3} \cdot 3 \mathrm{H}_{2} \mathrm{O}$ is unclear, but $\mathrm{InF}_{3} \cdot 3 \mathrm{H}_{2} \mathrm{O}$ is also a fluoride bridged

\footnotetext{
${ }^{a}$ Centre for Advanced Imaging, University of Queensland, Brisbane, Queensland 4072, Australia

${ }^{b}$ School of Chemistry, University of Southampton, Southampton SO17 1BJ, UK. E-mail: G.Reid@soton.ac.uk

${ }^{c}$ GE Healthcare UK, White Lion Road, Amersham HP7 9LL, UK

$\dagger$ Dedicated to the memory of Professor Ken Wade, FRS, in recognition of his many significant contributions to inorganic chemistry.

\$Electronic supplementary information (ESI) available: X-ray structure of $\left[\subset \mathrm{Me}_{2} \mathrm{~N}\left(\mathrm{CH}_{2}\right)_{2} \mathrm{NMe}\left(\mathrm{CH}_{2}\right)_{2}\right] \mathrm{Cl}$. CCDC 1053047-1053048 and 1053152-1053158. For ESI and crystallographic data in CIF or other electronic format see DOI: 10.1039/ c5dt01120e
}

polymer. ${ }^{2}$ Prolonged reflux of $\mathrm{GaF}_{3} \cdot 3 \mathrm{H}_{2} \mathrm{O}$ with pyridine in thf afforded the mer octahedral complex $\left[\mathrm{GaF}_{3}(\mathrm{py})_{3}\right]{ }^{3}$ whilst the reaction of $\mathrm{GaF}_{3} \cdot 3 \mathrm{H}_{2} \mathrm{O}$ with 1,4,7-tris(2-amino-3,5-di-tert-butylbenzyl)-1,4,7-triazacyclononane (L) under reflux conditions in ethanol, followed by crystallisation from $\mathrm{MeOH}$, afforded $\left[\mathrm{GaF}_{3}(\mathrm{~L})\right] \cdot 6 \mathrm{MeOH}$, as an $S_{6}$-symmetric $\mathrm{MeOH}$ hexamer. ${ }^{4} \mathrm{Com}-$ plexes of aluminium, gallium and indium fluorides with (neutral) $\mathrm{NH}_{3}$ ligands have been formed from the reaction of AlN or InN with $\mathrm{NH}_{4} \mathrm{~F}$ in supercritical ammonia at $400{ }^{\circ} \mathrm{C}$, from $\mathrm{NH}_{4} \mathrm{~F}$ or $\mathrm{NH}_{4} \mathrm{HF}_{2}$ and elemental $\mathrm{Ga}$, and from $\left[\mathrm{NH}_{4}\right]_{3}\left[\mathrm{MF}_{6}\right]$ and gaseous $\mathrm{NH}_{3}$, or $\mathrm{MF}_{3} \cdot 3 \mathrm{H}_{2} \mathrm{O}$ and liquid $\mathrm{NH}_{3} \cdot{ }^{5-9}$ The structures are usually polymeric with F-bridges. Studies in aqueous solution of various ethylenediamine derivatives of $\mathrm{Al}$ and $\mathrm{Ga}^{10,11}$ and of the indium fluoride-2,2'-bipyridyl- $\mathrm{H}_{2} \mathrm{O}-\mathrm{HF}$ system $^{12}$ have also been reported. The X-ray crystal structures of $\left[\operatorname{InF}_{3}\left(2,2^{\prime}\right.\right.$-bipy $\left.)\left(\mathrm{OH}_{2}\right)\right] \cdot 2 \mathrm{H}_{2} \mathrm{O}$ and $\left[\operatorname{InF}_{3}(\right.$ phen $\left.)\left(\mathrm{OH}_{2}\right)\right]$ obtained during the latter study have been described. ${ }^{12,13}$ The complexes $\left[\mathrm{MF}_{3}\left(4,4^{\prime}\right.\right.$-bipy) $]$ ( $\mathrm{M}=\mathrm{Ga}$ and $\left.\mathrm{In}\right)$ were formed hydrothermally from the respective $\mathrm{MF}_{3} \cdot 3 \mathrm{H}_{2} \mathrm{O}$ and $4,4^{\prime}$-bipyridyl in aqueous $\mathrm{HF}$ at $180^{\circ} \mathrm{C} .{ }^{14}$ Hydrothermal synthesis has also been used with other $\mathrm{N}$-donor ligands such as the macrocyclic $\mathrm{Me}_{3}$ tacn and BzMe $_{2}$-tacn $\left(\mathrm{Me}_{3}\right.$-tacn = 1,4,7-trimethyl-1,4,7-triazacyclononane, $\mathrm{BzMe}_{2}$-tacn = 1,4, dimethyl-7-benzyl-1,4,7-triaza- 
cyclononane, $\left.\mathrm{L}^{\prime}\right)$ to form $f a c-\left[\mathrm{MF}_{3}\left(\mathrm{~L}^{\prime}\right)\right] \cdot \mathrm{xH}_{2} \mathrm{O}$ for each of aluminium, gallium and indium. ${ }^{15}$ The compounds formed with $\mathrm{Me}_{3}$-tacn were found to crystallise as tetrahydrates, and extensive hydrogen bonding interactions between the water molecules of crystallisation and the coordinated fluorides were observed.

An alternative method for the production of the metalfluoro complexes is to first synthesise the metal chloride analogue and then perform a halide exchange reaction using reagents such as $\mathrm{Me}_{3} \mathrm{SiF}$ or $\mathrm{Me}_{3} \mathrm{SnF}$. For example, $\left[\mathrm{AlF}_{2}(\mathrm{py})_{4}\right] \mathrm{Cl}$ was successfully formed from the reaction of $\left[\operatorname{AlCl}_{3}(\mathrm{py})_{n}\right](n=1$ to 3) with $\mathrm{Me}_{3} \mathrm{SiF}$ in pyridine. ${ }^{16}$

The use of aluminium ${ }^{15}$ and gallium ${ }^{17}$ fluoride complexes incorporating ${ }^{18} \mathrm{~F}$ as a radiolabel, has attracted much recent interest as diagnostic imaging agents for PET (positron emission tomography). Key to their potential clinical suitability is the ability to incorporate the short-lived ${ }^{18} \mathrm{~F}$ isotope $\left(t_{1 / 2}=\right.$ $110 \mathrm{~min}$.) rapidly and cleanly in water; the resulting aluminium fluoride complexes are stable under physiological conditions,${ }^{15}$ while the gallium fluoride radio-product is stable in phosphate buffered saline (PBS) solution. ${ }^{17} \mathrm{~A}$ fuller understanding of the coordination chemistry of these Group 13 fluorides is expected to contribute to advancing the design of the next generation of ${ }^{18} \mathrm{~F}$ imaging agents.

Here we report on the systematic study of the preparation, spectroscopic and structural features of several series of complexes of the three Group 13 trifluorides with bi- and polydentate N-donor ligands, to explore the suitability of hydrothermal synthesis for other neutral ligands. The work also investigates the effect of replacing amine with neutral $\mathrm{N}$-heterocyclic ligands, and introducing the mer-trifluoride geometry, rather than the fac geometry present in the $\left[\mathrm{MF}_{3}\left(\mathrm{R}_{3}\right.\right.$-tacn $\left.)\right]$ systems.

\section{Experimental}

The $\mathrm{MF}_{3} \cdot 3 \mathrm{H}_{2} \mathrm{O}\left(\mathrm{M}=\mathrm{Al}\right.$, Ga or In), $\mathrm{GaCl}_{3}, 2,2^{\prime}$-bipyridyl, 1,10phenanthroline, 2,2':6',2"-terpyridyl, $4,4^{\prime}, 4^{\prime \prime}$-tris-t-butyl,$2,2^{\prime}: 6^{\prime}, 2^{\prime \prime}$-terpyridyl ( ${ }^{t} \mathrm{Bu}_{3}$-terpy), and $N, N, N^{\prime}, N^{\prime}, N^{\prime \prime}$-pentamethyldiethylenetriamine (PMDTA), were obtained from SigmaAldrich or Alfa-Aesar and used as supplied. Solvents were dried by distillation prior to use, $\mathrm{CH}_{2} \mathrm{Cl}_{2}$ and $\mathrm{CH}_{3} \mathrm{CN}$ from $\mathrm{CaH}_{2}$, hexane and toluene from sodium, THF from sodium benzophenone ketyl, methanol from magnesium/diiodine. All preparations of chloro-complexes were performed under an atmosphere of dry $\mathrm{N}_{2}$ using Schlenk techniques and spectroscopic samples were prepared in a dry, $\mathrm{N}_{2}$-purged glove box. Hydrothermal preparations were conducted in a $23 \mathrm{~mL}$ Teflon reactor vessel placed in a Parr stainless steel bomb. ${ }^{1} \mathrm{H}$ NMR spectra were recorded in $\mathrm{CD}_{3} \mathrm{OD}$ on a Bruker AVII 400 spectrometer and referenced to the residual proton resonance. ${ }^{19} \mathrm{~F}\left\{{ }^{1} \mathrm{H}\right\}$ NMR spectra were recorded in $\mathrm{CD}_{3} \mathrm{OD}$ on Bruker AVII 400 and DPX400 spectrometers, with $\mathrm{CFCl}_{3}$ as external reference. ${ }^{27} \mathrm{Al},{ }^{71} \mathrm{Ga}$ and ${ }^{115} \mathrm{In}$ NMR spectra were recorded in $\mathrm{CH}_{3} \mathrm{OH} /$ $\mathrm{CD}_{3} \mathrm{OD}$ on a Bruker AVII 400 NMR spectrometer and were refer- enced to $\left[\mathrm{Al}\left(\mathrm{OH}_{2}\right)_{6}\right]^{3+},\left[\mathrm{Ga}\left(\mathrm{OH}_{2}\right)_{6}\right]^{3+}$ and $\left[\mathrm{In}\left(\mathrm{OH}_{2}\right)_{6}\right]^{3+}$, respectively, in $\mathrm{H}_{2} \mathrm{O} / \mathrm{D}_{2} \mathrm{O}$ at $\mathrm{pH}=1$. Infra-red spectra were recorded as Nujol mulls between CsI plates on a Perkin Elmer Spectrum100 spectrometer over the range $4000-200 \mathrm{~cm}^{-1}$. Microanalyses were undertaken by London Metropolitan University.

\section{$\left[\mathrm{AlF}_{3}(\right.$ terpy) $] \cdot 3 \mathrm{H}_{2} \mathrm{O}$}

$\mathrm{AlF}_{3} \cdot 3 \mathrm{H}_{2} \mathrm{O}(0.100 \mathrm{~g}, 0.72 \mathrm{mmol})$ was suspended in freshly distilled water $(7 \mathrm{~mL})$ and terpy $(0.169 \mathrm{~g}, 0.72 \mathrm{mmol})$ was then added. The suspension was transferred into a Teflon container and loaded into a stainless steel high pressure vessel (Parr) and heated to $180^{\circ} \mathrm{C}$ for $15 \mathrm{~h}$. The vessel was then allowed to cool. A pale yellow solution had formed, a small aliquot of which was retained to grow crystals. For the remaining reaction mixture the solvent was removed in vacuo, yielding a pale orange solid. Yield: $0.18 \mathrm{~g}, 70 \%$. IR (Nujol, $\left.\nu / \mathrm{cm}^{-1}\right): 3370(\nu \mathrm{O}-$ $\mathrm{H}), 1640(\delta \mathrm{H}-\mathrm{O}-\mathrm{H}) 656,631,620(\mathrm{Al}-\mathrm{F}) .{ }^{1} \mathrm{H}$ NMR $(298 \mathrm{~K}): \delta=$ $8.90\left(\mathrm{~d}, J_{\mathrm{HH}} 8.0 \mathrm{~Hz},[2 \mathrm{H}], \mathrm{ArH},\right) 8.76\left(\mathrm{~d}, J_{\mathrm{HH}} 8.0 \mathrm{~Hz},[2 \mathrm{H}], \mathrm{ArH}\right)$, $8.54\left(\mathrm{~d}, J_{\mathrm{HH}} 8.0 \mathrm{~Hz},[2 \mathrm{H}], \mathrm{ArH}\right), 8.39\left(\mathrm{t}, J_{\mathrm{HH}} 8.0 \mathrm{~Hz},[2 \mathrm{H}], \mathrm{ArH}\right)$, $8.28\left(\mathrm{t}, J_{\mathrm{HH}} 8.0 \mathrm{~Hz},[\mathrm{H}], \mathrm{ArH}\right), 7.83\left(\mathrm{t}, J_{\mathrm{HH}} 6.0 \mathrm{~Hz},[2 \mathrm{H}], \mathrm{ArH}\right)$, $4.87\left(\mathrm{~s}, \mathrm{H}_{2} \mathrm{O}\right) \cdot{ }^{19} \mathrm{~F}\left\{{ }^{1} \mathrm{H}\right\}$ NMR $(298 \mathrm{~K}): \delta=-162.0\left(\mathrm{~d},{ }^{2} J_{\mathrm{FF}} 23 \mathrm{~Hz}\right.$, [2F]), $-177.3\left(\mathrm{t},{ }^{2} J_{\mathrm{FF}} 23 \mathrm{~Hz},[\mathrm{~F}]\right) .{ }^{27} \mathrm{Al} \mathrm{NMR}(298 \mathrm{~K}): \delta=16.7$ (br). Microanalyses on several batches, which were pure by spectroscopic analysis (including the single crystals), consistently gave $\mathrm{H}$ and $\mathrm{N}$ content as expected, but very variable (low) C content for this complex. Slow evaporation of the reaction solvent gave crystals suitable for X-ray diffraction.

\section{$\left[\mathrm{GaF}_{3}(\right.$ terpy $\left.)\right] \cdot 3 \mathrm{H}_{2} \mathrm{O}$}

Method 1: Method as for $\left[\mathrm{AlF}_{3}(\right.$ terpy $\left.)\right] \cdot 3 \mathrm{H}_{2} \mathrm{O}$ using $\mathrm{GaF}_{3} \cdot 3 \mathrm{H}_{2} \mathrm{O}$ $(0.200 \mathrm{~g}, 1.11 \mathrm{mmol})$ and terpy $(0.259 \mathrm{~g}, 1.11 \mathrm{mmol})$. Orange solid. Yield $0.366 \mathrm{~g}, 80 \%$. Required for $\mathrm{C}_{15} \mathrm{H}_{17} \mathrm{~F}_{3} \mathrm{GaN}_{3} \mathrm{O}_{3}$ (414.0): C, 43.5; H, 4.1; N, 10.2. Found: C, 43.3; H, 4.0; N, 10.3\%. IR (Nujol, $\left.\nu / \mathrm{cm}^{-1}\right): 3407(\nu \mathrm{O}-\mathrm{H}), 1644(\delta \mathrm{H}-\mathrm{O}-\mathrm{H}), 548$, 504, 490(sh) (Ga-F). ${ }^{1} \mathrm{H}$ NMR $(298 \mathrm{~K}): \delta=9.04\left(\mathrm{~d}, J_{\mathrm{HH}} 8.0 \mathrm{~Hz}\right.$, $[2 \mathrm{H}], \mathrm{ArH},) 8.80\left(\mathrm{~d}, J_{\mathrm{HH}} 8.0 \mathrm{~Hz},[2 \mathrm{H}], \mathrm{ArH}\right), 8.78\left(\mathrm{~d}, J_{\mathrm{HH}} 8.0 \mathrm{~Hz}\right.$, $[2 \mathrm{H}], \mathrm{ArH}), 8.64\left(\mathrm{t}, J_{\mathrm{HH}} 8.0 \mathrm{~Hz},[\mathrm{H}], \mathrm{ArH}\right), 8.49\left(\mathrm{t}, J_{\mathrm{HH}} 8.0 \mathrm{~Hz}\right.$, $[2 \mathrm{H}], \mathrm{ArH}), 8.03\left(\mathrm{t}, J_{\mathrm{HH}} 6.0 \mathrm{~Hz},[2 \mathrm{H}], \mathrm{ArH}\right), 4.87\left(\mathrm{~s}, \mathrm{H}_{2} \mathrm{O}\right)$. ${ }^{19} \mathrm{~F}\left\{{ }^{1} \mathrm{H}\right\}$ NMR (298 K): $\delta=-156.3(\mathrm{~s},[2 \mathrm{~F}]),-189.7$ (s, [F]); (183 K) $\delta=-154.5(\mathrm{~s},[2 \mathrm{~F}]),-188.9(\mathrm{~s},[\mathrm{~F}])$. Slow evaporation of the reaction solvent gave crystals suitable for X-ray diffraction.

Method 2: A suspension of $\left[\mathrm{GaCl}_{3}\right.$ (terpy)] (0.06 g, $0.15 \mathrm{mmol}$ ) in anhydrous MeCN $(5 \mathrm{~mL})$ was treated with $0.45 \mathrm{~mL}(0.45 \mathrm{mmol})$ of a $1.0 \mathrm{M}$ solution of $\left[\mathrm{NBu}_{4}\right] \mathrm{F}$ in thf. Addition of the fluoride source resulted in the dissolution of the chloride precursor and the formation of a pale yellow solution. The mixture was stirred at room temperature for $1 \mathrm{~h}$ and then the volatiles were removed in vacuo to yield a yellow gum. This was dissolved in a minimum volume of $\mathrm{CH}_{2} \mathrm{Cl}_{2}(\mathrm{ca} .2 \mathrm{~mL})$ and layered with hexane. A pale yellow precipitate formed overnight. Yield $0.042 \mathrm{~g}, 67 \%$. Spectroscopic data matched that observed for Method 1 and recrystallisation from $\mathrm{CH}_{2} \mathrm{Cl}_{2}$ / hexane yielded small crystals whose unit cell dimensions matched those of the crystals obtained via Method 1.

Method 3: $\left[\mathrm{GaCl}_{3}\right.$ (terpy) $](0.020 \mathrm{~g}, 0.050 \mathrm{mmol})$ was suspended in anhydrous MeCN $(5 \mathrm{~mL})$. A solution of $[\mathrm{K}(2.2 .2-$ 
crypt)]F (0.067 g, $0.150 \mathrm{mmol})$ in $3 \mathrm{~mL}$ anhydrous MeCN was added dropwise to the chloride precursor. Addition of the fluoride source resulted in the dissolution of the chloride precursor and the formation of a colourless solution. The mixture was stirred at room temperature for $1 \mathrm{~h}$, then the volatiles were removed in vacuo to give a white solid containing both the expected fluoride complex and the $[\mathrm{K}(2.2 .2$-crypt)]Cl by-product. Spectroscopic data for the former matched that observed from Method 1. No further purification was undertaken.

\section{$\left[\{\mathbf{G a}(\text { terpy }) \mathrm{F}\}_{2}(\boldsymbol{\mu}-\mathrm{F})_{2}\right]\left[\mathrm{PF}_{\mathbf{6}}\right]_{2} \cdot \mathbf{4} \mathbf{H}_{\mathbf{2}} \mathrm{O}$}

$\left[\mathrm{GaF}_{3}(\right.$ terpy $\left.)\right] \cdot 3 \mathrm{H}_{2} \mathrm{O}(0.013 \mathrm{~g}, 0.025 \mathrm{mmol})$ was dissolved in a mixture $\mathrm{CH}_{3} \mathrm{CN} / \mathrm{H}_{2} \mathrm{O}$ (ratio $1: 3,1.5 \mathrm{~mL}$ ) and added to $\left[\mathrm{NH}_{4}\right.$ $\left[\mathrm{PF}_{6}\right](0.004 \mathrm{~g}, 0.025 \mathrm{mmol})$ in $\mathrm{H}_{2} \mathrm{O}(1.5 \mathrm{~mL})$. The reaction solution was left to evaporate slowly at room temperature, resulting in the formation of orange crystals suitable for single crystal X-ray diffraction. Required for $\mathrm{C}_{30} \mathrm{H}_{30} \mathrm{~F}_{16} \mathrm{Ga}_{2} \mathrm{~N}_{6} \mathrm{O}_{4} \mathrm{P}_{2}$ : C, 34.5; $\mathrm{H}$, 2.9; N, 8.1. Found: C, 34.4; H, 2.9; N, 8.2\%. ${ }^{19} \mathrm{~F}\left\{{ }^{1} \mathrm{H}\right\}$ NMR $\left(\mathrm{CD}_{3} \mathrm{OD}, 183 \mathrm{~K}\right): \delta=-75.1\left(\mathrm{~d},\left[\mathrm{PF}_{6}\right]^{-}\right),-156.4(\mathrm{~s},[2 \mathrm{~F}]),-189.8$ (s, [F]).

\section{$\left[\operatorname{InF}_{3}(\right.$ terpy) $] \cdot 3 \mathbf{H}_{2} \mathrm{O}$}

Method as for $\left[\mathrm{AlF}_{3}(\right.$ terpy $] \cdot 3 \mathrm{H}_{2} \mathrm{O}$ using $\mathrm{InF}_{3} \cdot 3 \mathrm{H}_{2} \mathrm{O}(0.100 \mathrm{~g}$, $0.44 \mathrm{mmol})$ and terpy $(0.1032 \mathrm{~g}, 0.44 \mathrm{mmol})$. Pale orange solid. Yield: $0.10 \mathrm{~g}, 49 \%$. Required for $\mathrm{C}_{15} \mathrm{H}_{17} \mathrm{~F}_{3} \mathrm{InN}_{3} \mathrm{O}_{3}$ (459.1): C, 39.2; H, 3.7; N, 9.2. Found: C, 39.4; H, 3.9; N, 9.3\%. IR (Nujol, $\left.\nu / \mathrm{cm}^{-1}\right): 3490,3400(\nu \mathrm{O}-\mathrm{H}), 1654(\delta \mathrm{H}-\mathrm{O}-\mathrm{H}), 476,427,406$ (InF). ${ }^{1} \mathrm{H}$ NMR $(298 \mathrm{~K}): \delta=8.68(\mathrm{~m},[2 \mathrm{H}], \mathrm{ArH}), 8.61\left(\mathrm{~d}, J_{\mathrm{HH}} 8.0\right.$ $\mathrm{Hz},[2 \mathrm{H}], \mathrm{ArH}), 8.39$ (d, $\left.J_{\mathrm{HH}} 8.0 \mathrm{~Hz},[2 \mathrm{H}], \mathrm{ArH}\right), 8.07-7.97$ (m, [3H], ArH), 7.47 (m, [2H], ArH), 4.87 (s, H $\left.\mathrm{H}_{2} \mathrm{O}\right) .{ }^{19} \mathrm{~F}\left\{{ }^{1} \mathrm{H}\right\}$ NMR (298 K): $\delta=-157.8$ (br s [2F]), -181.6 (br s [F]); (183 K): -157.5 (br s $[2 \mathrm{~F}]$ ), -178.5 (br s [F]).

\section{$\left[\mathrm{AlF}_{3}(\mathrm{bipy})\left(\mathrm{OH}_{2}\right)\right] \cdot \mathbf{2} \mathrm{H}_{2} \mathrm{O}$}

Method as for $\left[\mathrm{AlF}_{3}\right.$ (terpy)] $3 \mathrm{H}_{2} \mathrm{O}$ using $\mathrm{AlF}_{3} \cdot 3 \mathrm{H}_{2} \mathrm{O}(0.200 \mathrm{~g}$, $1.45 \mathrm{mmol})$ and bipy $(0.226 \mathrm{~g}, 1.45 \mathrm{mmol})$. The resulting pale pink solid was washed with acetonitrile and filtered to leave a white solid which was dried in vacuo. Yield: $0.20 \mathrm{~g}, 47 \%$. Required for $\mathrm{C}_{10} \mathrm{H}_{14} \mathrm{AlF}_{3} \mathrm{~N}_{2} \mathrm{O}_{3}$ (294.2): C, 40.8; H, 4.8; N, 9.5. Found: C, 40.6; H, 4.6; N, 9.7\%. IR (Nujol, $\nu / \mathrm{cm}^{-1}$ ): 3533, 3422 $(\nu \mathrm{O}-\mathrm{H}), 1689 \mathrm{br}(\delta \mathrm{H}-\mathrm{O}-\mathrm{H}), 610,590(\mathrm{sh})(\mathrm{Al}-\mathrm{F}) .{ }^{1} \mathrm{H}$ NMR $\left(\mathrm{CD}_{3} \mathrm{OD}, 298 \mathrm{~K}\right): \delta=9.04\left(\mathrm{~d}, J_{\mathrm{HH}} 4.0 \mathrm{~Hz},[1 \mathrm{H}], \mathrm{ArH}\right), 8.76\left(\mathrm{~d}, J_{\mathrm{HH}}\right.$ $4.4 \mathrm{~Hz},[1 \mathrm{H}], \mathrm{ArH}), 8.59$ (d, $\left.J_{\mathrm{HH}} 7.6 \mathrm{~Hz},[1 \mathrm{H}], \mathrm{ArH}\right), 8.44$ (d, $J_{\mathrm{HH}}$ $8.1 \mathrm{~Hz},[1 \mathrm{H}], \mathrm{ArH}), 8.33\left(\mathrm{t}, J_{\mathrm{HH}} 7.7 \mathrm{~Hz},[1 \mathrm{H}], \mathrm{ArH}\right), 8.14\left(\mathrm{t}, J_{\mathrm{HH}}\right.$ $7.5 \mathrm{~Hz},[1 \mathrm{H}], \mathrm{ArH}), 7.81-7.85(\mathrm{~m},[1 \mathrm{H}], \mathrm{ArH}), 7.63\left(\mathrm{t}, J_{\mathrm{HH}} 5.9 \mathrm{~Hz}\right.$, [1H], ArH), $4.75\left(\mathrm{~s}, \mathrm{H}_{2} \mathrm{O}\right) .{ }^{19} \mathrm{~F}\left\{{ }^{1} \mathrm{H}\right\} \mathrm{NMR}(298 \mathrm{~K}): \delta=-172.8$ (br s); $(183 \mathrm{~K}):-162.3(\mathrm{~s},[\mathrm{~F}]),-176.4(\mathrm{~s},[2 \mathrm{~F}]) .{ }^{27} \mathrm{Al} \mathrm{NMR}(298 \mathrm{~K}): \delta=$ $8.3\left(\mathrm{br} \mathrm{s}, w_{1 / 2}=400 \mathrm{~Hz}\right.$ ), resonance lost on cooling below $233 \mathrm{~K}$. Slow evaporation of the reaction solvent gave crystals suitable for X-ray diffraction.

\section{$\left[\mathrm{GaF}_{3}(\right.$ bipy $\left.)\left(\mathrm{OH}_{2}\right)\right] \cdot 2 \mathrm{H}_{2} \mathrm{O}$}

Method as for $\left[\mathrm{AlF}_{3}\right.$ (terpy) $] \cdot 3 \mathrm{H}_{2} \mathrm{O}$ using $\mathrm{GaF}_{3} \cdot 3 \mathrm{H}_{2} \mathrm{O}(0.200 \mathrm{~g}$, $1.11 \mathrm{mmol})$ and bipy (0.158 g, $1.11 \mathrm{mmol})$. Pale pink solid. Yield $0.314 \mathrm{~g}, 84 \%$. Required for $\mathrm{C}_{10} \mathrm{H}_{14} \mathrm{~F}_{3} \mathrm{GaN}_{2} \mathrm{O}_{3}$ (337.0): C, 35.7; H, 4.1; N, 8.3. Found: C, 35.6; H, 4.1; N, 8.2\%. IR (Nujol, $\left.\nu / \mathrm{cm}^{-1}\right): 3500,3380(\nu \mathrm{O}-\mathrm{H}), 1660$ br $(\delta \mathrm{H}-\mathrm{O}-\mathrm{H}), 527,473(\mathrm{Ga}-\mathrm{F})$. ${ }^{1} \mathrm{H}$ NMR (CD $\left.\mathrm{CD}, 298 \mathrm{~K}\right): \delta=9.07$ (br, [2H], ArH), 8.66 (d, $\left.J_{\mathrm{HH}} 8 \mathrm{~Hz},[2 \mathrm{H}], \mathrm{ArH}\right), 8.37\left(\mathrm{t}, J_{\mathrm{HH}} \sim 8 \mathrm{~Hz},[2 \mathrm{H}], \operatorname{ArH}\right), 7.89(\mathrm{~s}$, [2H] ArH); (183 K): 9.09 (s, [2H]), 8.81 (br, s [2H], ArH), 8.47 (s, $[2 \mathrm{H}]), 7.97$ (s, [2H]), $4.75\left(\mathrm{~s}, \mathrm{H}_{2} \mathrm{O}\right) .{ }^{19} \mathrm{~F}\left\{{ }^{1} \mathrm{H}\right\}$ NMR $(298 \mathrm{~K}): \delta=$ -176.2 (br s); (183 K): -160.9 (s, [F]), -181.5 (s, [2F]). Slow evaporation of the reaction solvent gave crystals suitable for $\mathrm{X}$-ray diffraction.

\section{$\left[\operatorname{InF}_{3}(\right.$ bipy $\left.)\left(\mathrm{OH}_{2}\right)\right] \cdot 2 \mathrm{H}_{2} \mathrm{O}$}

Method as for $\left[\mathrm{AlF}_{3}\right.$ (terpy)] $3 \mathrm{H}_{2} \mathrm{O}$ using $\operatorname{InF}_{3} \cdot 3 \mathrm{H}_{2} \mathrm{O}(0.200 \mathrm{~g}$, $0.89 \mathrm{mmol})$ and bipy $(0.138 \mathrm{~g}, 0.89 \mathrm{mmol})$, yielding a pale yellow solid. Yield: $0.17 \mathrm{~g}, 50 \%$. Required for $\mathrm{C}_{10} \mathrm{H}_{14} \mathrm{~F}_{3} \mathrm{InN}_{2} \mathrm{O}_{3}$ (382.1): C, 31.4; H, 3.7; N, 7.3. Found: C, 31.6; H, 3.6; N, 7.4\%. IR (Nujol, $\left.\nu / \mathrm{cm}^{-1}\right)$ : 3422 br $(\nu \mathrm{O}-\mathrm{H}), 1655$ br $(\delta \mathrm{H}-\mathrm{O}-\mathrm{H}), 442$, 428, 404 (In-F). ${ }^{1} \mathrm{H}$ NMR ( $\left.\mathrm{CD}_{3} \mathrm{OD}, 298 \mathrm{~K}\right): \delta=8.78$ (br s, [2H], ArH), 8.68 (br s, [2H], ArH), 8.45 (br s, [2H], ArH), 7.96 (br s, [2H], ArH), 4.75 (s, $\mathrm{H}_{2} \mathrm{O}$ ); (183 K): 8.99 (br, s), 8.95 (br s,), 8.66 (s), 8.52 (vbr), 8.05-7.99 (m). $\left.{ }^{19} \mathrm{~F}^{1}{ }^{1} \mathrm{H}\right\}$ NMR ( $\left.\mathrm{CD}_{3} \mathrm{OD}, 298 \mathrm{~K}\right)$ : not observed; (183 K): $\delta=-152.9(\mathrm{~s},[\mathrm{~F}]),-178.1$ (s, [2F]).

\section{$\left[\mathrm{AlF}_{3}(\right.$ phen $\left.)\left(\mathrm{OH}_{2}\right)\right]$}

Method as for $\left[\mathrm{AlF}_{3}\right.$ (terpy) $\left.\left(\mathrm{OH}_{2}\right)\right] \cdot 2 \mathrm{H}_{2} \mathrm{O}$, but using $\mathrm{AlF}_{3} \cdot 3 \mathrm{H}_{2} \mathrm{O}$ $(0.200 \mathrm{~g}, 1.45 \mathrm{mmol})$ and phen $(0.261 \mathrm{~g}, 1.45 \mathrm{mmol})$. White solid. Yield: $0.254 \mathrm{~g}, 62 \%$. Required for $\mathrm{C}_{12} \mathrm{H}_{10} \mathrm{AlF}_{3} \mathrm{~N}_{2} \mathrm{O}$ (282.2): C, 51.1; H, 3.6; N, 9.9. Found: C, 50.9; H, 3.4; N, 10.0\%. IR (Nujol, $\left.\nu / \mathrm{cm}^{-1}\right)$ : 3200 br $(\nu \mathrm{O}-\mathrm{H}), 1670(\delta \mathrm{H}-\mathrm{O}-\mathrm{H}), 637,608(\mathrm{Al}-\mathrm{F})$. ${ }^{1} \mathrm{H}$ NMR (298 K): $\delta=9.30\left(\mathrm{~d}, J_{\mathrm{HH}} 5.1 \mathrm{~Hz},[1 \mathrm{H}], \operatorname{ArH}\right), 9.17$ (d, $\left.J_{\mathrm{HH}} 3.7 \mathrm{~Hz},[1 \mathrm{H}], \mathrm{ArH}\right), 8.88\left(\mathrm{~d}, J_{\mathrm{HH}} 7.6 \mathrm{~Hz},[1 \mathrm{H}], \mathrm{ArH}\right), 8.66$ (d, $\left.J_{\mathrm{HH}} 8.8 \mathrm{~Hz},[1 \mathrm{H}], \mathrm{ArH}\right), 8.25$ (s, [1H], ArH), 8.14 (m, [1H], ArH), 8.09 (s, [1H], ArH), $7.93\left(\mathrm{~m}, J_{\mathrm{HH}} 4.2 \mathrm{~Hz},[1 \mathrm{H}], \mathrm{ArH}\right), 4.75$ (s, $\mathrm{H}_{2} \mathrm{O}$ ). ${ }^{19} \mathrm{~F}\left\{{ }^{1} \mathrm{H}\right\} \operatorname{NMR}(298 \mathrm{~K}): \delta=-157.3$ (br s $\left.[\mathrm{F}]\right),-174.8$ (s, [2F]); (183 K): $\delta=-162.5$ (br s [F]), -177.3 (s, [2F]);. ${ }^{27} \mathrm{Al} \mathrm{NMR}$ (298 K): $\delta=8.8\left(\right.$ br s, $\left.w_{1 / 2} 450 \mathrm{~Hz}\right)$.

\section{$\left[\mathrm{GaF}_{3}(\right.$ phen $\left.)\left(\mathrm{OH}_{2}\right)\right]$}

Method as for $\left[\mathrm{AlF}_{3}(\right.$ terpy) $] \cdot 3 \mathrm{H}_{2} \mathrm{O}$, using $\mathrm{GaF}_{3} \cdot 3 \mathrm{H}_{2} \mathrm{O}(0.200 \mathrm{~g}$, $1.11 \mathrm{mmol})$ and phen $(0.200 \mathrm{~g}, 1.11 \mathrm{mmol})$. Pale red solid. Yield $0.315 \mathrm{~g}, 79 \%$. Required for $\mathrm{C}_{12} \mathrm{H}_{10} \mathrm{~F}_{3} \mathrm{GaN}_{2} \mathrm{O}$ (324.9): C, 44.4; H, 3.1; N, 8.6. Found: C, 44.4; H, 3.1; N, 8.5\%. IR (Nujol, $\left.\nu / \mathrm{cm}^{-1}\right): 3200$ br $(\nu \mathrm{O}-\mathrm{H}), 1680(\delta \mathrm{H}-\mathrm{O}-\mathrm{H}), 560,543,511(\mathrm{Ga}-\mathrm{F})$. ${ }^{1} \mathrm{H}$ NMR $(298 \mathrm{~K}): \delta=9.34\left(\mathrm{~d}, J_{\mathrm{HH}} 4 \mathrm{~Hz},[2 \mathrm{H}], \mathrm{ArH}\right), 8.89(\mathrm{~d}$, $\left.J_{\mathrm{HH}} 12 \mathrm{~Hz},[2 \mathrm{H}], \mathrm{ArH}\right), 8.26$ (s, [2H], ArH), 8.15 (br, [2H], ArH) 4.87 (s, $\left.\mathrm{H}_{2} \mathrm{O}\right)$; (183 K): 9.34 (s), 9.02 (d), 8.94 (d), 8.33-8.21 (m). ${ }^{19} \mathrm{~F}\left\{{ }^{1} \mathrm{H}\right\}$ NMR (298 K): $\delta=-152$ (br s [F]), -176 (br, [2F]); (183 K): $\delta=-151.0$ (br s [F]), -184.0 (s, [2F]). Slow evaporation of the reaction solvent gave crystals suitable for X-ray diffraction.

\section{$\left[\operatorname{InF}_{3}(\right.$ phen $\left.)\left(\mathrm{OH}_{2}\right)\right]$}

Method as for $\left[\mathrm{AlF}_{3}(\right.$ terpy) $] \cdot 3 \mathrm{H}_{2} \mathrm{O}$, but using $\operatorname{InF}_{3} \cdot 3 \mathrm{H}_{2} \mathrm{O}$ (0.200 g, $0.89 \mathrm{mmol}$ ) and phen $(0.160 \mathrm{~g}, 0.89 \mathrm{mmol})$. Pale orange solid. Yield: $0.16 \mathrm{~g}, 49 \%$. Required for $\mathrm{C}_{12} \mathrm{H}_{10} \mathrm{~F}_{3} \mathrm{InN}_{2} \mathrm{O}$ (370.0): C, 39.0; H, 2.7; N, 7.6. Found: C, 39.2; H, 2.8; N, 7.7\%. IR (Nujol, $\left.\nu / \mathrm{cm}^{-1}\right)$ : $3360 \mathrm{br}, 3177(\nu \mathrm{O}-\mathrm{H}), 1660 \mathrm{w}(\delta \mathrm{H}-\mathrm{O}-\mathrm{H})$, 446, 422 br, 405(sh) (In-F). ${ }^{1} \mathrm{H}$ NMR (298 K): $\delta=9.30$ (br s, 
[1H], ArH), 9.02-8.96 (br [3H], ArH), 8.29-8.07 (br, m, [4H], $\mathrm{ArH}), 4.87$ (s, $\left.\mathrm{H}_{2} \mathrm{O}\right)$; (183 K): 9.28 (s), 8.98 (s), 8.76 (s), 8.33-8.22 (m), $7.95(\mathrm{~m}) .{ }^{19} \mathrm{~F}\left\{{ }^{1} \mathrm{H}\right\}$ NMR (298 K): not observed; (183 K): $\delta=$ -157 (br, [F]), -199 (br, [2F]).

\section{$\left[\mathrm{GaCl}_{3}(\right.$ terpy $\left.)\right]$}

A solution of terpy $(0.258 \mathrm{~g}, 1.11 \mathrm{mmol})$ in anhydrous $\mathrm{CH}_{2} \mathrm{Cl}_{2}$ $(5 \mathrm{~mL})$ was added dropwise to a solution of $\mathrm{GaCl}_{3}(0.195 \mathrm{~g}$, $1.11 \mathrm{mmol}$ ) in $5 \mathrm{~mL}$ anhydrous $\mathrm{CH}_{2} \mathrm{Cl}_{2}$. A white precipitate formed upon addition of the ligand. The mixture was stirred at room temperature for $1 \mathrm{~h}$. The product was isolated by filtration, washed with hexane and dried in vacuo. White solid. Yield: $0.402 \mathrm{~g}, 88 \%$. Required for $\mathrm{C}_{15} \mathrm{H}_{11} \mathrm{Cl}_{3} \mathrm{GaN}_{3}$ : C, 44.0; $\mathrm{H}$, 2.7; N, 10.3. Found: C, 43.5; H, 2.6; N, 10.1\%. IR (Nujol, $\nu / \mathrm{cm}^{-1}$ ): $267 \mathrm{~s}, 254 \mathrm{br},(\mathrm{Ga}-\mathrm{Cl}) .{ }^{1} \mathrm{H}$ NMR $\left(\mathrm{CD}_{3} \mathrm{CN}, 298 \mathrm{~K}\right): \delta=9.06-9.04$ $(\mathrm{m},[2 \mathrm{H}], \mathrm{ArH}), 8.75-8.73(\mathrm{~m},[3 \mathrm{H}], \mathrm{ArH}), 8.67-8.65(\mathrm{~m},[2 \mathrm{H}]$, ArH), 8.53-8.49 (m, [2H], ArH), 8.08-8.05 (m, [2H], ArH).

\section{$\left[\mathrm{GaCl}_{3}\left({ }^{t} \mathrm{Bu}_{3}\right.\right.$-terpy)}

A solution of ${ }^{t} \mathrm{Bu}_{3}$-terpy $(0.111 \mathrm{~g}, 0.278 \mathrm{mmol})$ in anhydrous $\mathrm{CH}_{2} \mathrm{Cl}_{2}(5 \mathrm{~mL})$ was added dropwise to a solution of $\mathrm{GaCl}_{3}$ $(0.025 \mathrm{~g}, 0.139 \mathrm{mmol})$ in anhydrous $\mathrm{CH}_{2} \mathrm{Cl}_{2}(5 \mathrm{~mL})$. Addition of the ligand resulted in the formation of a white precipitate. The mixture was stirred at room temperature for $1 \mathrm{~h}$. The solid was isolated by filtration, washed with a further $10 \mathrm{~mL} \mathrm{CH} \mathrm{Cl}_{2}$ and dried in vacuo. White solid. Yield $0.062 \mathrm{~g}, 77 \%$. Required for $\mathrm{C}_{27} \mathrm{H}_{35} \mathrm{Cl}_{3} \mathrm{GaN}_{3} \cdot \mathrm{CH}_{2} \mathrm{Cl}_{2}$ (662.60): C, 50.7; H, 5.6; N, 6.3. Found: C, 50.4; H, 5.6; N, 6.5\%. ${ }^{1} \mathrm{H}$ NMR ( $\left.\mathrm{CD}_{3} \mathrm{CN}, 298 \mathrm{~K}\right): \delta=$ 1.42 (s, [18H] , $\left.{ }^{t} \mathrm{Bu}\right), 1.44\left(\mathrm{~s},[9 \mathrm{H}],{ }^{t} \mathrm{Bu}\right), 7.47-7.46$ (br m, $[2 \mathrm{H}]$, ArH), 8.50 (br s, [2H], ArH), 8.61-8.60 (br m, [2H], ArH), 8.77 (br s, [2H], ArH).

The same reaction performed in a $1: 1$ stoichiometric ratio gave a spectroscopically identical product. However, crystals formed from the filtrate gave the ionic by-product $\left[\mathrm{GaCl}_{2}\left({ }^{t} \mathrm{Bu}_{3}-\right.\right.$ terpy) $]\left[\mathrm{GaCl}_{4}\right] \cdot \mathrm{CH}_{2} \mathrm{Cl}_{2}$.

\section{$\left[\subset \mathrm{Me}_{2} \mathrm{~N}\left(\mathrm{CH}_{2}\right)_{2} \mathrm{NMe}\left(\mathrm{CH}_{2}\right)_{2}\right]_{2}\left[\mathrm{Al}_{2} \mathrm{~F}_{8}\left(\mathrm{OH}_{2}\right)_{2}\right] \cdot 2 \mathrm{H}_{2} \mathrm{O}$}

Method as for $\left[\mathrm{AlF}_{3}\right.$ (bipy) $\left.\left(\mathrm{OH}_{2}\right)\right] \cdot 2 \mathrm{H}_{2} \mathrm{O}$, but using $\mathrm{AlF}_{3} \cdot 3 \mathrm{H}_{2} \mathrm{O}$ (0.200 g, $1.45 \mathrm{mmol})$ and PMDTA (0.3 mL, $1.45 \mathrm{mmol})$. A brown gum was obtained after removing the solvent. This was washed with $\mathrm{MeCN}$, producing a white powder. Yield: $0.44 \mathrm{~g}$, 58\%. Required for $\mathrm{C}_{14} \mathrm{H}_{42} \mathrm{Al}_{2} \mathrm{~F}_{8} \mathrm{~N}_{4} \mathrm{O}_{4}$ (536.4): C, 31.4; $\mathrm{H}, 7.8 ; \mathrm{N}$, 10.4. Found: C, $31.3 ; \mathrm{H}, 7.8 ; \mathrm{N}, 10.4 \%$. IR (Nujol, $\left.\nu / \mathrm{cm}^{-1}\right): 3370$ br ( $\nu$ O-H), 1673 ( $\delta$ H-O-H), 616 br, 569 br, (Al-F). ${ }^{1} \mathrm{H}$ NMR $\left(\mathrm{CD}_{3} \mathrm{CN}, 298 \mathrm{~K}\right): \delta=4.87\left(\mathrm{~s}, \mathrm{H}_{2} \mathrm{O}\right), 3.35\left(\mathrm{t}, J_{\mathrm{HH}} 6.0 \mathrm{~Hz},[4 \mathrm{H}]\right.$, $\mathrm{CH}_{2}$ ), 3.07 (s, [6H], Me), 2.67 (br s, [4H], $\mathrm{CH}_{2} \mathrm{CH}_{2}$ ), 2.32 (s, $[3 \mathrm{H}], \mathrm{Me}) \cdot{ }^{19} \mathrm{~F}\left\{{ }^{1} \mathrm{H}\right\} \mathrm{NMR}\left(\mathrm{CD}_{3} \mathrm{CN}, 298 \mathrm{~K}\right): \delta=-194.6$ (6 lines $\left.{ }^{1} J_{\mathrm{AlF}}=38 \mathrm{~Hz}\right) \cdot{ }^{27} \mathrm{Al} \mathrm{NMR}\left(\mathrm{CH}_{3} \mathrm{CN}, 298 \mathrm{~K}\right): \delta=48.7$ (s). Slow evaporation of the reaction solvent gave crystals suitable for X-ray diffraction.

The corresponding gallium and indium fluoride reactions were conducted similarly.

\section{$\left[\subset \mathrm{Me}_{2} \mathrm{~N}\left(\mathrm{CH}_{2}\right)_{2} \mathrm{NMe}\left(\mathrm{CH}_{2}\right)_{2}\right]_{2}\left[\mathrm{Ga}_{2} \mathrm{~F}_{8}\left(\mathrm{OH}_{2}\right)_{2}\right] \cdot 2 \mathrm{H}_{2} \mathrm{O}$}

Required for $\mathrm{C}_{14} \mathrm{H}_{42} \mathrm{~F}_{8} \mathrm{Ga}_{2} \mathrm{~N}_{4} \mathrm{O}_{4}$ (619.9): C, 27.0; H, 6.8; N, 9.0. Found: C, 26.7; H, 6.4; N, 9.8\%. ${ }^{1} \mathrm{H}$ NMR $\left(\mathrm{CD}_{3} \mathrm{CN}, 298 \mathrm{~K}\right): \delta=$ $4.87\left(\mathrm{~s}, \mathrm{H}_{2} \mathrm{O}\right), 3.35$ (t, $\left.J_{\mathrm{HH}} 6.0 \mathrm{~Hz},[4 \mathrm{H}], \mathrm{CH}_{2}\right), 3.07$ (s, [6H], Me), 2.67 (br s, [4H], $\mathrm{CH}_{2} \mathrm{CH}_{2}$ ), 2.32 (s, [3H], Me). ${ }^{13} \mathrm{C}\left\{{ }^{1} \mathrm{H}\right\} \mathrm{NMR}$ $\left(\mathrm{CD}_{3} \mathrm{CN}\right): \delta=44.18(\mathrm{~s}), 44.84(\mathrm{~s}), 48.04$ (s), 61.59 (s). IR (Nujol, $\left.\nu / \mathrm{cm}^{-1}\right)$ : 3420 br $(\nu \mathrm{O}-\mathrm{H}), 1673(\delta \mathrm{H}-\mathrm{O}-\mathrm{H}), 518 \mathrm{br}$, $477 \mathrm{br},(\mathrm{Ga}-\mathrm{F})$.

A small number of crystals obtained from the $\mathrm{Ga}$ and In reaction mixtures were found to be $\left[\subset \mathrm{Me}_{2} \mathrm{~N}\left(\mathrm{CH}_{2}\right)_{2} \mathrm{NMe}\left(\mathrm{CH}_{2}\right)_{2}\right] \mathrm{Cl}$ (see text and ESI†).

\section{X-Ray experimental}

Details of the crystallographic data collection and refinement parameters are given in Table 1 . Crystals suitable for single crystal X-ray analysis were obtained as described above. Data collections used a Rigaku AFC12 goniometer equipped with an enhanced sensitivity (HG) Saturn724+ detector mounted at the window of an FR-E+ SuperBright molybdenum $(\lambda=0.71073 \AA)$ rotating anode generator with VHF Varimax optics $(70 \mu \mathrm{m}$ focus) with the crystal held at $100 \mathrm{~K}\left(\mathrm{~N}_{2}\right.$ cryostream). Structure solution and refinements were performed with either SHELX (S/L)97 or SHELX(S/L) $2013^{18}$ and were straightforward, except where detailed below. $\mathrm{H}$ atoms bonded to $\mathrm{C}$ were placed in calculated positions using the default $\mathrm{C}-\mathrm{H}$ distance and refined using a riding model. In the case of the $\left[\mathrm{AlF}_{3}(\right.$ bipy $\left.)\left(\mathrm{OH}_{2}\right)\right] \cdot 2 \mathrm{H}_{2} \mathrm{O}$ structure, the $\mathrm{H}$-atoms on the co-crystallised water molecules were not located in the difference map. While not included in the refinement, the $\mathrm{H}$-atoms are inferred from the $\mathrm{H}$-bonding distances of $\mathrm{F} \cdots \mathrm{O}$ and $\mathrm{O} \cdots \mathrm{O}$ and are thus included in the formulae. The $\mathrm{H}$-atoms on both the coordinated and co-crystallised water molecules could not be located in the structure of $\left[C \mathrm{Me}_{2} \mathrm{~N}\left(\mathrm{CH}_{2}\right)_{2} \mathrm{NMe}\left(\mathrm{CH}_{2}\right)_{2}\right]_{2}\left[\mathrm{Al}_{2} \mathrm{~F}_{8}\left(\mathrm{OH}_{2}\right)_{2}\right] \cdot 2 \mathrm{H}_{2} \mathrm{O}$. While not included in the refinement, the $\mathrm{H}$-atoms are similarly inferred and included in the formulae. CCDC numbers 1053047-1053048 and 1053152-1053158.

\section{Results and discussion}

The unreactive and poorly soluble nature of the $\mathrm{MF}_{3} \cdot 3 \mathrm{H}_{2} \mathrm{O}$ makes reaction with neutral ligands in organic solvents difficult or impossible. We therefore used the hydrothermal approach $\left(180{ }^{\circ} \mathrm{C} / 15 \mathrm{~h}\right)$ and found this gave high yields of $\left[\mathrm{MF}_{3}\right.$ (terpy) $] \cdot 3 \mathrm{H}_{2} \mathrm{O}$ (below). The same approach was then extended to reactions with $N, N, N^{\prime}, N^{\prime}, N^{\prime \prime}$-pentamethyldiethylenetriamine (PMDTA), and to the diimines, 2,2'-bipyridyl and 1,10-phenanthroline.

\section{$\left[\mathrm{MF}_{3}(\right.$ terpy $\left.)\right] \cdot 3 \mathrm{H}_{2} \mathrm{O}(\mathbf{M}=\mathbf{A l}, \mathbf{G a}$ or In $)$}

The reaction of the $\mathrm{MF}_{3} \cdot 3 \mathrm{H}_{2} \mathrm{O}$ with terpy in a $1: 1$ molar ratio in water at $180{ }^{\circ} \mathrm{C} / 15 \mathrm{~h}$, followed by removal of the solvent in vacuo at room temperature, gave high yields of the $\left[\mathrm{MF}_{3}-\right.$ (terpy)] $3 \mathrm{H}_{2} \mathrm{O}$ as pale orange solids. Slow evaporation of a small portion of the mother liquor at ambient temperatures gave crystals of $\left[\mathrm{MF}_{3}(\right.$ terpy $\left.)\right] \cdot 3 \mathrm{H}_{2} \mathrm{O}(\mathrm{M}=\mathrm{Al}$ or $\mathrm{Ga})$. For $\mathrm{M}=\mathrm{Al}$, the structure shows (Fig. 1) a distorted octahedral geometry about the aluminium, the distortions being largely due to the rigid terpy ligand which results in $\mathrm{N}-\mathrm{Al}-\mathrm{N}$ angles significantly less 
Table 1 Crystallographic parameters ${ }^{a}$

\begin{tabular}{|c|c|c|c|c|}
\hline Compound & {$\left[\mathrm{AlF}_{3}(\right.$ terpy $\left.)\right] \cdot 3 \mathrm{H}_{2} \mathrm{O}$} & {$\left[\mathrm{GaF}_{3}(\right.$ terpy $\left.)\right] \cdot 3 \mathrm{H}_{2} \mathrm{O}$} & {$\left[\mathrm{AlF}_{3}(\right.$ bipy $\left.)\left(\mathrm{OH}_{2}\right)\right] \cdot 2 \mathrm{H}_{2} \mathrm{O}$} & {$\left[\mathrm{GaF}_{3}(\right.$ bipy $\left.)\left(\mathrm{OH}_{2}\right)\right] \cdot 2 \mathrm{H}_{2} \mathrm{O}$} \\
\hline Formula & $\mathrm{C}_{15} \mathrm{H}_{17} \mathrm{AlF}_{3} \mathrm{~N}_{3} \mathrm{O}_{3}$ & $\mathrm{C}_{15} \mathrm{H}_{17} \mathrm{~F}_{3} \mathrm{GaN}_{3} \mathrm{O}_{3}$ & $\mathrm{C}_{10} \mathrm{H}_{14} \mathrm{AlF}_{3} \mathrm{~N}_{2} \mathrm{O}_{3}$ & $\mathrm{C}_{10} \mathrm{H}_{14} \mathrm{~F}_{3} \mathrm{GaN}_{2} \mathrm{O}_{3}$ \\
\hline$M$ & 371.30 & 414.04 & 294.20 & 336.95 \\
\hline Space group (no.) & $P 2_{1} / c(14)$ & $P 2_{1} / c(14)$ & $P 2_{1} / n(14)$ & $P 2_{1} / n(14)$ \\
\hline$a / \AA$ & $9.4806(9)$ & $10.323(5)$ & $8.976(5)$ & $9.056(3)$ \\
\hline$b / \AA$ & $25.104(2)$ & $9.708(5)$ & $7.300(5)$ & $7.371(2)$ \\
\hline$c / \AA$ & $7.0846(6)$ & $16.131(5)$ & $19.062(5)$ & $18.904(6)$ \\
\hline$\gamma /{ }^{\circ}$ & 90 & 90 & 90 & 90 \\
\hline$U / \AA^{3}$ & $1633.0(3)$ & $1589.6(12)$ & $1246.9(11)$ & $1259.6(7)$ \\
\hline$Z$ & 4 & 4 & 4 & 4 \\
\hline$\mu\left(\mathrm{Mo}-\mathrm{K}_{\alpha}\right) / \mathrm{mm}^{-1}$ & 0.177 & 1.785 & 0.207 & 2.228 \\
\hline$F(000)$ & 768 & 840 & 608 & 680 \\
\hline Total number reflections & 6803 & 9623 & 8257 & 11425 \\
\hline$R_{1}, \mathrm{w} R_{2}$ (all data) & $0.146,0.172$ & $0.056,0.089$ & $0.104,0.168$ & $0.051,0.132$ \\
\hline Compound & {$\left[\mathrm{GaF}_{3}(\right.$ phen $\left.)-\left(\mathrm{OH}_{2}\right)\right]$} & $\begin{array}{l}{\left[\mathrm{GaCl}_{2}\left({ }^{t} \mathrm{Bu}_{3} \text {-terpy) }\right]-\right.} \\
{\left[\mathrm{GaCl}_{4}\right] \cdot \mathrm{CH}_{2} \mathrm{Cl}_{2}}\end{array}$ & $\begin{array}{l}{\left[\subset \mathrm{Ce}_{2} \mathrm{~N}\left(\mathrm{CH}_{2}\right)_{2} \mathrm{NMe}-\right.} \\
\left.\left(\mathrm{CH}_{2}\right)_{2}\right]_{2}\left[\mathrm{Al}_{2} \mathrm{~F}_{8}\left(\mathrm{OH}_{2}\right)_{2}\right] \cdot 2 \mathrm{H}_{2} \mathrm{O}\end{array}$ & $\begin{array}{l}{\left[\{\mathrm{Ga}(\text { terpy }) \mathrm{F}\}_{2}(\mu-\mathrm{F})_{2}\right]-} \\
{\left[\mathrm{PF}_{6}\right]_{2} \cdot 4 \mathrm{H}_{2} \mathrm{O}}\end{array}$ \\
\hline Formula & $\mathrm{C}_{12} \mathrm{H}_{10} \mathrm{~F}_{3} \mathrm{GaN}_{2} \mathrm{O}$ & $\mathrm{C}_{14} \mathrm{H}_{18.5} \mathrm{Cl}_{4} \mathrm{GaN}_{1.5}$ & $\mathrm{C}_{14} \mathrm{H}_{42} \mathrm{Al}_{2} \mathrm{~F}_{8} \mathrm{~N}_{4} \mathrm{O}_{4}$ & $\mathrm{C}_{30} \mathrm{H}_{30} \mathrm{~F}_{16} \mathrm{Ga}_{2} \mathrm{~N}_{6} \mathrm{O}_{4} \mathrm{P}_{2}$ \\
\hline$M$ & 324.94 & 419.32 & 538.4 & 1043.98 \\
\hline Crystal system & Monoclinic & Triclinic & Monoclinic & Triclinic \\
\hline Space group (no.) & $P 2_{1} / n(14)$ & $P \overline{1}(2)$ & $C 2 / c(15)$ & $P \overline{1}(2)$ \\
\hline$a / \AA$ & $8.3906(5)$ & $11.674(3)$ & $24.054(10)$ & $9.774(2)$ \\
\hline$b / \AA$ & $7.1940(5)$ & $12.642(4)$ & $7.558(3)$ & $10.460(2)$ \\
\hline$c / \AA$ & $19.0124(13)$ & $13.756(5)$ & $16.389(7)$ & $10.646(2)$ \\
\hline$\alpha /{ }^{\circ}$ & 90 & $89.137(17)$ & 90 & $78.064(12)$ \\
\hline$\beta /{ }^{\circ}$ & $100.631(3)$ & $78.682(16)$ & $123.374(4)$ & $62.736(8)$ \\
\hline No. of parameters, restraints & 178,2 & 379,0 & 170,6 & 271,0 \\
\hline$R_{1}, \mathrm{w} R_{2}[I>2 \sigma(I)]^{b}$ & $0.025,0.064$ & $0.086,0.192$ & $0.078,0.212$ & $0.043,0.109$ \\
\hline$R_{1}, \mathrm{w} R_{2}$ (all data) & $0.028,0.066$ & $0.125,0.211$ & $0.098,0.231$ & $0.049,0.111$ \\
\hline
\end{tabular}

than $90^{\circ} / 180^{\circ}$, whereas the $\mathrm{F}-\mathrm{Al}-\mathrm{F}$ and (cis) $\mathrm{F}-\mathrm{Al}-\mathrm{N}$ angles are close to those expected for an octahedron.

There is extensive $\mathrm{H}$-bonding between the fluoride ligands and the lattice water molecules (Fig. 2), as well as $\pi$-stacking of the aromatic rings (Fig. 3).

The Al-F bond lengths are very similar to those observed in fac-[ $\mathrm{AlF}_{3}\left(\mathrm{Me}_{3}\right.$-tacn $\left.)\right] \cdot 4 \mathrm{H}_{2} \mathrm{O},{ }^{15}$ but the $\mathrm{Al}-\mathrm{N}$ bonds are shorter by $\sim 0.05 \AA$, suggesting the macrocyclic ring may limit the close approach of the $\mathrm{N}$ atoms to the metal centre. The molecular structure of $\left[\mathrm{GaF}_{3}\right.$ (terpy) $] \cdot 3 \mathrm{H}_{2} \mathrm{O}$ is very similar to that of the $\mathrm{Al}(\mathrm{III})$ complex (Fig. 4) with the Ga-F bonds ca. 0.1 A longer than the corresponding $\mathrm{Al}-\mathrm{F}$, whilst the $\mathrm{Ga}-\mathrm{N}$ and $\mathrm{Al}-\mathrm{N}$ are little different.

As found in the aluminium complex, the structure of $\left[\mathrm{GaF}_{3}(\right.$ terpy) $] \cdot 3 \mathrm{H}_{2} \mathrm{O}$ shows extensive $\mathrm{H}$-bonding as shown in
Fig. 5 as well as $\pi$-stacking interactions of 3.57 and $3.62 \AA$ (see ESI Fig. S1 $\$$ ), although the arrangements differ in detail. The Ga-F distances are very similar to those found in fac$\left[\mathrm{GaF}_{3}\left(\mathrm{Me}_{3}\right.\right.$-tacn $\left.)\right] \cdot 4 \mathrm{H}_{2} \mathrm{O} .{ }^{15}$ The Ga-N distances are also not significantly different to those found in the $\left[\mathrm{GaX}_{3}\right.$ (terpy) $](\mathrm{X}=\mathrm{Cl}$ or $\mathrm{Br}){ }^{19}$ although the extensive H-bonding in the fluoride complex is absent in the structures of the heavier halides. The single bond covalent radii of $\mathrm{Al}(\mathrm{III})$ and $\mathrm{Ga}(\mathrm{III})$ are quoted in standard texts as nearly identical $(\sim 1.25 \AA)$, although the ionic radius of $\mathrm{Ga}^{3+}$ is $\sim 0.07 \AA$ larger than that of $\mathrm{Al}^{3+} \cdot{ }^{20}$ The limited number of structurally characterised complexes of the trifluorides limits detailed comparisons, but it seems that the metaldonor bond length may be very sensitive to the electronegativity of the donor atom, with little difference between $\mathrm{Al}-\mathrm{L}$ 


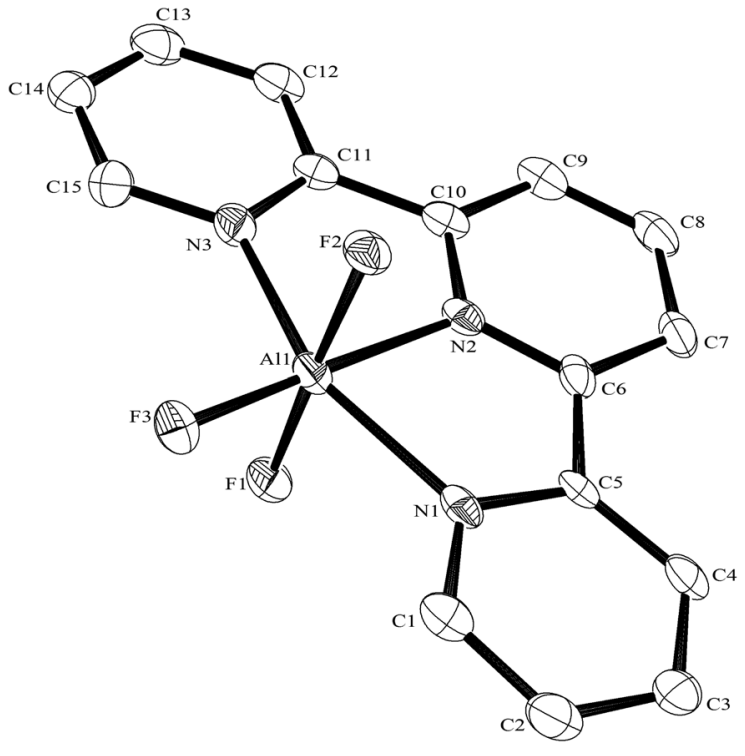

Fig. 1 The structure of the Al species in $\left[\mathrm{AlF}_{3}(\right.$ terpy) $] \cdot 3 \mathrm{H}_{2} \mathrm{O}$ with ellipsoids drawn at the $50 \%$ probability level. Hydrogen atoms on terpy are omitted for clarity. Selected bond lengths $(\AA)$ and angles $\left(^{\circ}\right)$ are Al1-F1 = 1.761(2), Al1-F2 = 1.762(2), Al1-F3 = 1.737(2), Al1-N1 = 2.057(3), Al1-N2 = 2.010(3), Al1-N3 = 2.059(3), F1-Al1-F3 = 92.55(12), F2-Al1-F3 = 91.08(11), F1-Al1-F2 = 176.34(13), N1-Al1-N2 = 77.58(13), N2-Al1-N3 = 78.17(13), N1-Al1-N3 = 155.73(13).

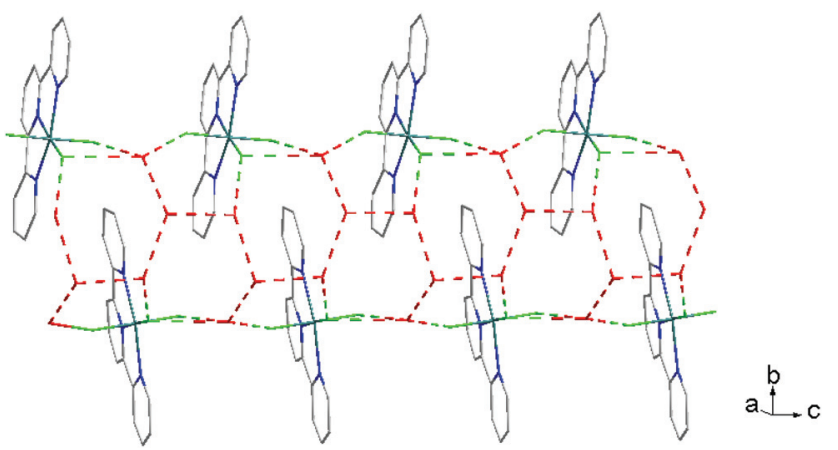

Fig. 2 The $\mathrm{H}$-bonding network (red) in the crystals of $\left[\mathrm{AlF}_{3}\left(\right.\right.$ terpy)] $33 \mathrm{H}_{2} \mathrm{O}$.

and $\mathrm{Ga}-\mathrm{L}$ with heavier donor ligands and halides, ${ }^{21}$ but significant differences in the M-F bond lengths. The effects of solvent molecules and hydrogen bonding also complicates the interpretation of small differences in metal-donor bond lengths, ${ }^{19,22}$ and these may be a significant factor in the extensively hydrogen bonded metal fluoride complexes.

Interpretation of the spectroscopic properties (Experimental section) of the three terpy complexes is straightforward. The IR spectra show strong, very broad features due to the $\nu(\mathrm{OH})$ and $\delta(\mathrm{HOH})$ modes of the water and three $\nu(\mathrm{MF})$ stretches, as expected for a mer-trifluoride (theory $2 \mathrm{~A}_{1}+\mathrm{B}_{1}$ ). The ${ }^{1} \mathrm{H}$ NMR spectra in $\mathrm{CD}_{3} \mathrm{OD}$ exhibit sharp multiplet resonances for the terpy protons and a broad signal for the water, whilst the

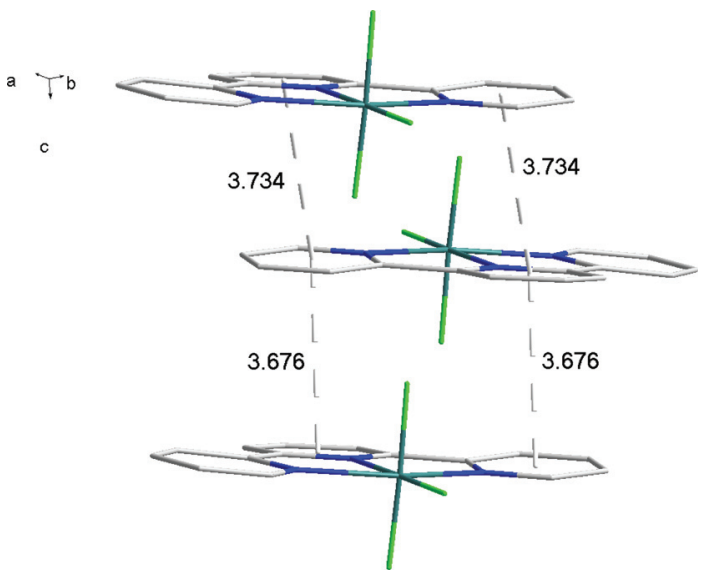

Fig. $3 \pi$-stacking in the structure of $\left[\mathrm{AlF}_{3}(\right.$ terpy) $] \cdot 3 \mathrm{H}_{2} \mathrm{O}$ with lattice water omitted for clarity.

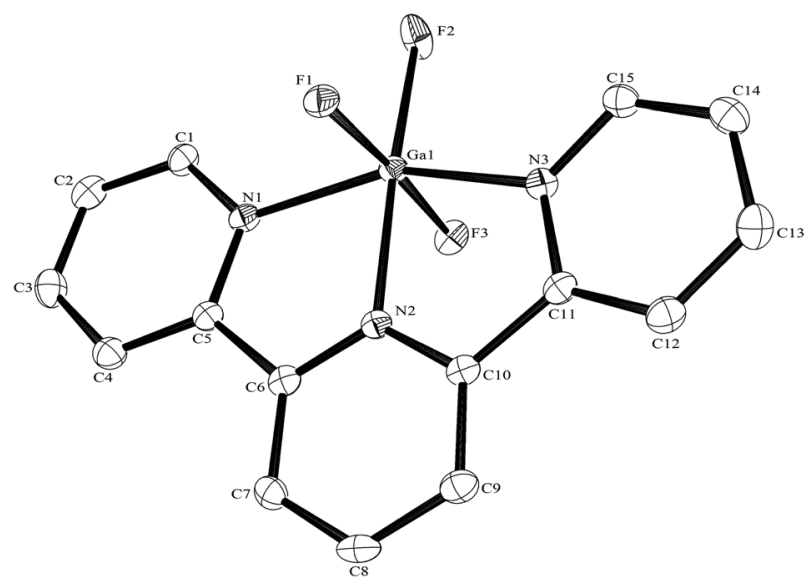

Fig. 4 The structure of the $\mathrm{Ga}$ species in $\left[\mathrm{GaF}_{3}(\right.$ terpy) $] \cdot 3 \mathrm{H}_{2} \mathrm{O}$ with ellipsoids drawn at the $50 \%$ probability level. Hydrogen atoms are omitted for clarity. Selected bond lengths $(\AA)$ and angles $\left(^{\circ}\right): G a 1-F 1=1.8781(17)$, Ga1-F2 = 1.8780(16), Ga1-F3 = 1.8895(17), Ga1-N1 = 2.082(2), Ga1-N2 = 2.045(2), Ga1-N3 = 2.093(2), F1-Ga1-F2 = 92.12(7), F2-Ga1-F3 = 90.60(7), F1-Ga1-F3 = 176.29(7), N1-Ga1-N2 = 77.21(8), N2-Ga1-N3 = 77.26(9), N1-Ga1-N3 = 154.28(8).

${ }^{19} \mathrm{~F}\left\{{ }^{1} \mathrm{H}\right\}$ NMR spectra show two resonances in a $2: 1$ ratio due to $\mathrm{F}_{\text {trans } \mathrm{F}}$ and $\mathrm{F}_{\text {trans } \mathrm{N}}$ respectively. The ${ }^{19} \mathrm{~F}\left\{{ }^{1} \mathrm{H}\right\}$ resonances for the $\left[\mathrm{AlF}_{3}\right.$ (terpy)] show doublet and triplet ${ }^{2} J_{\mathrm{FF}}$ couplings of 23 $\mathrm{Hz}$, but for the gallium and indium complexes only broad singlets are observed over the temperature range 298-183 K. ${ }^{23}$ The aluminium complex also exhibited a ${ }^{27} \mathrm{Al}$ NMR resonance at $\delta=16.7$ as a broad singlet with no resolved ${ }^{1} J_{\mathrm{AlF}}$ coupling, which is in the range expected for six-coordinate aluminium. ${ }^{24}$ Neither the gallium nor indium complex exhibited a metal nucleus resonance, probably due to fast quadrupolar relaxation. The multinuclear NMR data show that the molecular structures of these complexes are maintained in $\mathrm{MeOH}$ solution. 


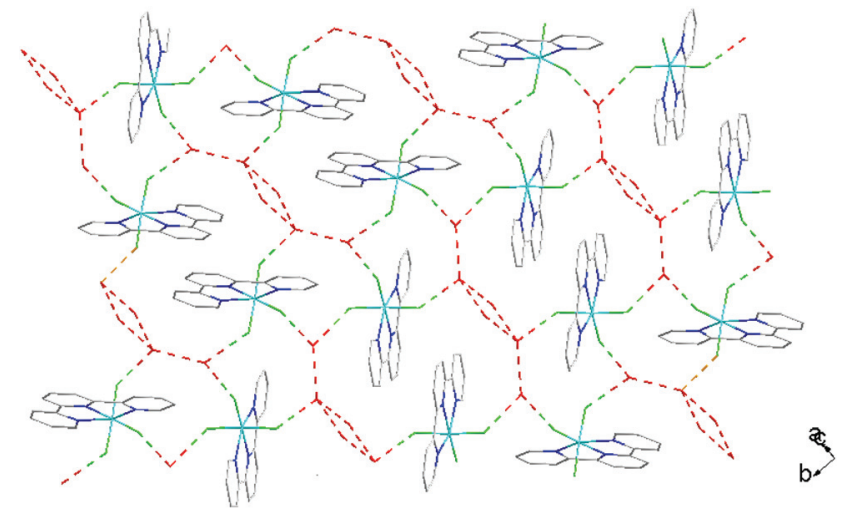

Fig. 5 View showing the $\mathrm{H}$-bonding network (red) in $\left[\mathrm{GaF}_{3}\right.$ (terpy)].3 $\mathrm{H}_{2} \mathrm{O}$.

The synthesis of the $\left[\mathrm{GaF}_{3}(\right.$ terpy) $]$ by halide exchange was also explored. The $\left[\mathrm{GaCl}_{3}\right.$ (terpy)] precursor was made in high yield by reaction of $\mathrm{GaCl}_{3}$ with terpy in anhydrous $\mathrm{CH}_{2} \mathrm{Cl}_{2}$ solution, and its composition confirmed by microanalysis and spectroscopically. Complete fluorination of $\left[\mathrm{GaCl}_{3}\right.$ (terpy)] in MeCN solution was achieved at room temperature with $\left[{ }^{n} \mathrm{Bu}_{4} \mathrm{~N}\right] \mathrm{F}$ in thf or $[\mathrm{K}(2.2 .2$-crypt $)] \mathrm{F}$ in $\mathrm{MeCN}$; the $\left[\mathrm{GaF}_{3}\right.$ (terpy)] produced was spectroscopically identical to samples produced by the hydrothermal route, and crystals obtained using the $\left[{ }^{n} \mathrm{Bu}_{4} \mathrm{~N}\right] \mathrm{F}$ route had the same unit cell. Fluorination with $[\mathrm{K}(2.2 .2$-crypt $)] \mathrm{F}$ was also successful at $80{ }^{\circ} \mathrm{C}$ in $\mathrm{MeCN}$, confirming the gallium species is thermally stable under these conditions. The $\left[\mathrm{GaCl}_{3}(\right.$ terpy) $]$ was relatively poorly soluble in organic solvents and in an attempt to increase the solubility, the corresponding complex of $4,4^{\prime}, 4^{\prime \prime}$-tris-t-butyl,-2,2':6',2"-terpyridyl, $\left[\mathrm{GaCl}_{3}\left({ }^{t} \mathrm{Bu}_{3}\right.\right.$-terpy) $)$, was prepared. Its properties were very similar to the terpy complex (Experimental section), but it was in fact rather less soluble in common organic solvents. However, crystals grown from the filtrate from one synthesis were found to be $\left[\mathrm{GaCl}_{2}\left({ }^{t} \mathrm{Bu}_{3}\right.\right.$-terpy) $)\left[\mathrm{GaCl}_{4}\right] \cdot \mathrm{CH}_{2} \mathrm{Cl}_{2}$, containing a five-coordinate cation. This minor by-product probably results from the presence of a small excess of $\mathrm{GaCl}_{3}$ in the synthesis, which extracts a chloride from the neutral species to form the stable $\left[\mathrm{GaCl}_{4}\right]^{-}$anion. The structure of the cation is shown in Fig. 6. The geometry is a distorted trigonal bipyramid with apical $\mathrm{N}$, distorted by the steric constraints of the ${ }^{t} \mathrm{Bu}_{3}$ terpy (N3-Ga1-N1 $\left.=155.1(2)^{\circ}\right)$, as also found in the neutral $\left[\mathrm{GaCl}_{3}(\right.$ terpy) $]$ complex above. Comparisons of the bond lengths between $\left[\mathrm{GaCl}_{2}\left({ }^{t} \mathrm{Bu}_{3} \text {-terpy) }\right]^{+}\right.$and $\left[\mathrm{GaCl}_{3}\right.$ (terpy) $\left.)\right],{ }^{19}$ as expected, show shorter bonds in the five-coordinate cation $(\mathrm{Ga}-\mathrm{Cl}=2.169(2), 2.195(2), \mathrm{Ga}-\mathrm{N}=1.995(5)-2.092(5) \AA) \mathrm{com}-$ pared to the six-coordinate neutral complex $(\mathrm{Ga}-\mathrm{Cl}=2.2511(5)$ $-2.4118(6), \mathrm{Ga}-\mathrm{N}=2.0412(5)-2.1024(15) \AA)$.

\section{$\left[\left\{\mathrm{Ga}\right.\right.$ (terpy) $\left.\mathrm{F}_{2}(\boldsymbol{\mu}-\mathrm{F})_{2}\right]\left[\mathrm{PF}_{6}\right]_{2} \cdot \mathbf{4} \mathrm{H}_{2} \mathrm{O}$}

The $\mathrm{fac}-\left[\mathrm{GaF}_{3}\left(\mathrm{BzMe}_{2}\right.\right.$-tacn)$]$ has been shown to function as a neutral 'metalloligand' through the coordinated fluorides towards alkali metal and ammonium cations in aqueous solu-

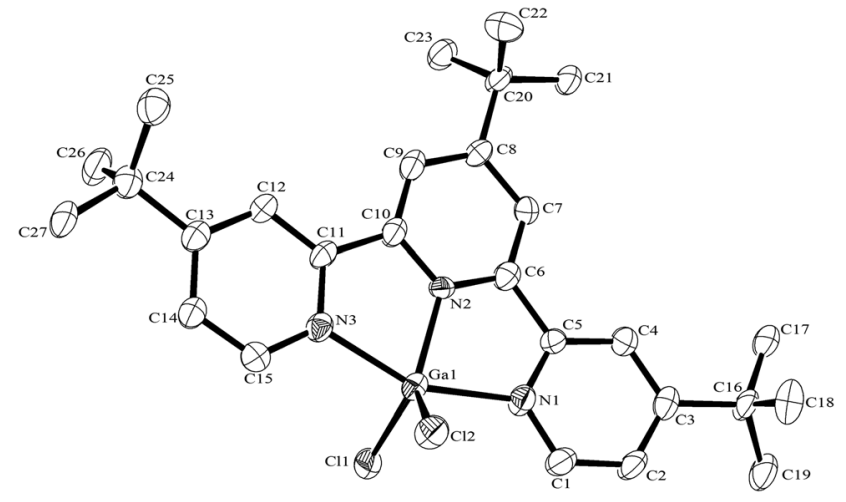

Fig. 6 The structure of the cation in $\left[\mathrm{GaCl}_{2}\left({ }^{\mathrm{t}} \mathrm{Bu}_{3}\right.\right.$-terpy) $]\left[\mathrm{GaCl}_{4}\right]$ with ellipsoids drawn at the $50 \%$ probability level. Hydrogen atoms are omitted for clarity. Selected bond lengths $(\AA \AA)$ and angles $\left({ }^{\circ}\right)$ : Ga1-N1 = 2.092(5), Ga1-N2 = 1.995(5), Ga1-N3 = 2.066(5), Ga1-Cl1 = 2.195(2), Ga1-Cl2 = 2.169(2), N2-Ga1-N3 = 77.9(2), N1-Ga1-N2 = 77.3(2), N1Ga1-N3 = 155.1(2), N2-Ga1-Cl2 = 125.68(18), N3-Ga1-Cl2 = 97.76(17), $\mathrm{N} 1-\mathrm{Ga} 1-\mathrm{Cl} 2=95.78(17), \mathrm{N} 2-\mathrm{Ga} 1-\mathrm{Cl} 1=120.20(18), \mathrm{N} 3-\mathrm{Ga} 1-\mathrm{Cl}=$ 96.80(16), N1-Ga1-Cl1 = 96.56(18), Cl1-Ga1-Cl2 = 114.10(8).

tion, leading to supramolecular arrays with Ga-F-M linkages, ${ }^{25}$ whilst combination of $\mathrm{Gd}^{3+}$ and $f a c-\left[\mathrm{GaF}_{3}\left(\mathrm{Me}_{3}\right.\right.$-tacn)] leads to ' $\left[\mathrm{Gd}_{3} \mathrm{Ga}_{2}\right]$ ' cores that are of interest as molecular magnets. ${ }^{26} \mathrm{~A}$ key feature of these systems is that the three facial Ga-F bonds remain intact throughout. To explore if the meridional trifluoride arrangement present in $\left[\mathrm{GaF}_{3}(\right.$ terpy $\left.)\right]$ could function in a similar manner, a $\mathrm{H}_{2} \mathrm{O}-\mathrm{MeCN}$ solution containing $\left[\mathrm{GaF}_{3}-\right.$ (terpy)] and $\left[\mathrm{NH}_{4}\right]\left[\mathrm{PF}_{6}\right]$ was allowed to evaporate slowly. Orange crystals formed and an X-ray structure analysis on these showed that rather than forming an ammonium-metalloligand complex, the dimer, $\left[\left\{\mathrm{Ga}\right.\right.$ (terpy) $\left.\mathrm{F}_{2}(\mu-\mathrm{F})_{2}\right]\left[\mathrm{PF}_{6}\right]_{2} \cdot 4 \mathrm{H}_{2} \mathrm{O}$ had formed.

The centrosymmetric cation (Fig. 7) contains six-coordinate gallium, severely distorted from regular octahedral by the steric constraints of the terpy ligand $\left(\mathrm{N} 1-\mathrm{Ga} 1-\mathrm{N} 3=153.85(10)^{\circ}\right)$, and the fluoride bridges are asymmetric $(\mathrm{Ga} 1-\mathrm{F} 1=1.889(2)$, Ga1-F1a $=2.003(2) \AA$ ). There is extensive hydrogen bonding linking the lattice water molecules, the cations and the $\left[\mathrm{PF}_{6}\right]^{-}$ anions (Fig. 8). The complex, formed by dissociation of one fluoride from each gallium centre, followed by dimerisation, dissolves in $\mathrm{CD}_{3} \mathrm{OD}$ with decomposition and formation of a white precipitate. The ${ }^{19} \mathrm{~F}\left\{{ }^{1} \mathrm{H}\right\}$ NMR spectrum of the supernatant shows only $\left[\mathrm{GaF}_{3}(\right.$ terpy $\left.)\right]$ and $\left[\mathrm{PF}_{6}\right]^{-}$as significant species.

\section{$\left[\mathrm{MF}_{3}(\right.$ diimine $\left.)\left(\mathrm{OH}_{2}\right)\right] \cdot n \mathrm{H}_{2} \mathrm{O}$}

The hydrothermal method also proved a suitable route to obtain $\left[\mathrm{MF}_{3}(\right.$ bipy $\left.)\left(\mathrm{OH}_{2}\right)\right] \cdot 2 \mathrm{H}_{2} \mathrm{O}$ and $\left[\mathrm{MF}_{3}(\right.$ phen $\left.)\left(\mathrm{OH}_{2}\right)\right]$ in good yields. The structures of $\left[\operatorname{InF}_{3}(\right.$ phen $\left.)\left(\mathrm{OH}_{2}\right)\right]$ and $\left[\operatorname{InF}_{3}(\right.$ bipy $)$ $\left.\left(\mathrm{OH}_{2}\right)\right] \cdot 2 \mathrm{H}_{2} \mathrm{O}$ obtained from samples made from aqueous HF solution have been reported previously. ${ }^{13}$ The $\left[\mathrm{MF}_{3}\right.$ (bipy)$\left.\left(\mathrm{OH}_{2}\right)\right] \cdot 2 \mathrm{H}_{2} \mathrm{O}(\mathrm{M}=\mathrm{Al}$ or $\mathrm{Ga})$ are monoclinic, $P 2_{1} / n$, with sixcoordinate metal centres and a mer-fluoride arrangement, 


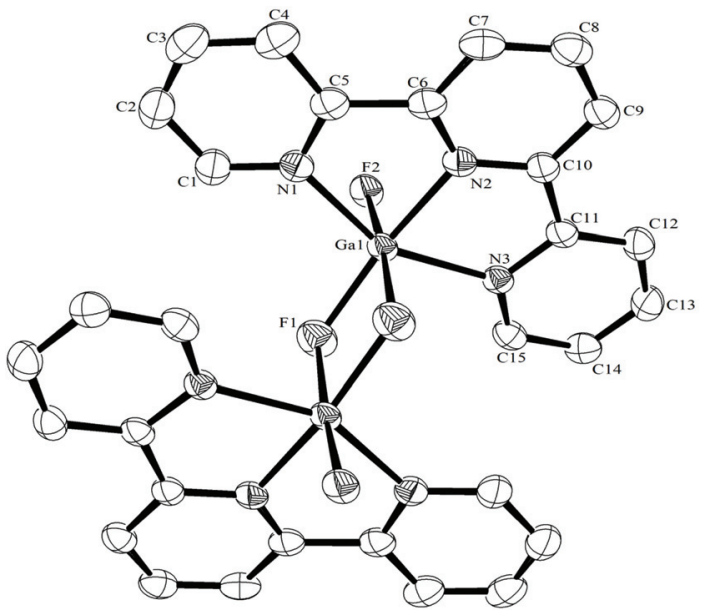

Fig. 7 The structure of the centrosymmetric cation in $\left[\{G a(t e r p y) F\}_{2}-\right.$ $\left.(\mu-\mathrm{F})_{2}\right]\left[\mathrm{PF}_{6}\right]_{2} \cdot 4 \mathrm{H}_{2} \mathrm{O}$ with ellipsoids drawn at the $50 \%$ probability level. Hydrogen atoms are omitted for clarity. Selected bond lengths $(\AA)$ and angles $\left(^{\circ}\right)$ : Ga1-F2 = 1.8564(17), Ga1-F1 = 1.889(2), Ga1-F1a = 2.003(2), Ga1$\mathrm{N} 2=2.026(2), \mathrm{Ga} 1-\mathrm{N} 1=2.095(3), \mathrm{Ga1}-\mathrm{N} 32.110(2), \mathrm{F} 2-\mathrm{Ga} 1-\mathrm{F} 1=98.39(8)$, $\mathrm{F} 2-\mathrm{Ga} 1-\mathrm{F} 1 \mathrm{a}=174.58(7), \mathrm{F} 1-\mathrm{Ga} 1-\mathrm{F} 1 \mathrm{a}=76.19(9), \mathrm{F} 2-\mathrm{Ga} 1-\mathrm{N} 2=$ 97.02(8), F1-Ga1-N2 = 88.40(9), F2-Ga1-N1 = 88.84(9), F1a-Ga-N1 = 102.47(9), F1-Ga1-N1 = 92.37(9), N2-Ga1-N1 = 77.26(10), F2-Ga1-N3 = 88.19(8), F1a-Ga1-N3 = 103.67(9), F1-Ga1-N3 = 93.03(9), N2-Ga1-N3 = 77.34(9), N1-Ga1-N3 = 153.85(10), Ga1-F1-Ga1a = 103.81(9).

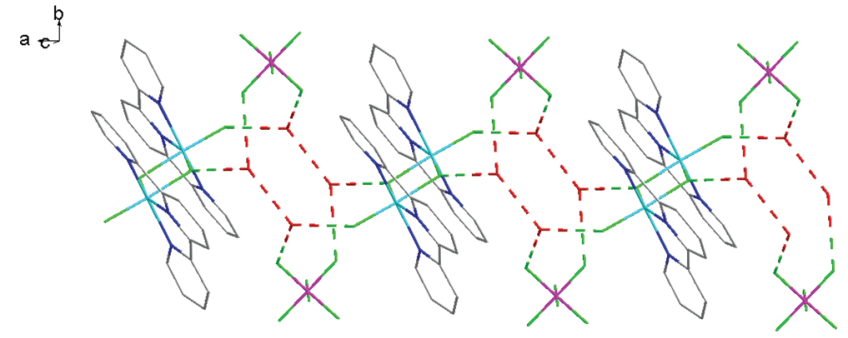

Fig. 8 View showing the $\mathrm{H}$-bonding network (red) in $\left[\{\mathrm{Ga} \text { (terpy) }\}_{2^{-}}\right.$ $\left.(\mu-\mathrm{F})_{2}\right]\left[\mathrm{PF}_{6}\right]_{2} \cdot 4 \mathrm{H}_{2} \mathrm{O}$.

which means the neutral bipy ligand is trans to $\mathrm{OH}_{2} / \mathrm{F}$ (Fig. 9 and 10).

For $\left[\mathrm{InF}_{3}(\mathrm{bipy})\left(\mathrm{OH}_{2}\right)\right] \cdot 2 \mathrm{H}_{2} \mathrm{O},{ }^{13} \mathrm{In}-\mathrm{F}_{\text {trans } \mathrm{F}}=2.065(2), 2.070(2)$, In $-\mathrm{F}_{\text {trans } \mathrm{N}}=2.002(3), \mathrm{In}-\mathrm{N}_{\text {trans } \mathrm{F}}=2.260(4)$, In $-\mathrm{N}_{\text {trans } \mathrm{O}}=2.243(4)$, In-O $=2.130(4) \AA$. Comparing the bond lengths in the three compounds reveals some interesting trends. The $\mathrm{M}-\mathrm{N}$ distances are only slightly different $(\leq 0.02 \AA)$ between $\mathrm{Al}$ and $\mathrm{Ga}$, whereas the $\mathrm{M}-\mathrm{F}$ and $\mathrm{M}-\mathrm{O}$ distances increase by between 0.06-0.1 $\AA$; as noted with the terpy compounds above, the more electronegative donors have the greatest differences. The corresponding bond lengths all increase in the indium complex as expected, but here the differences with donor type are less clear, with all showing an increase of $\sim 0.15-0.2 \AA$ over the gallium analogue. In all of the complexes $\mathrm{H}$-bonding to the lattice water and $\pi$-stacking of the aromatic rings is also evident (Fig. 11-13 and S2

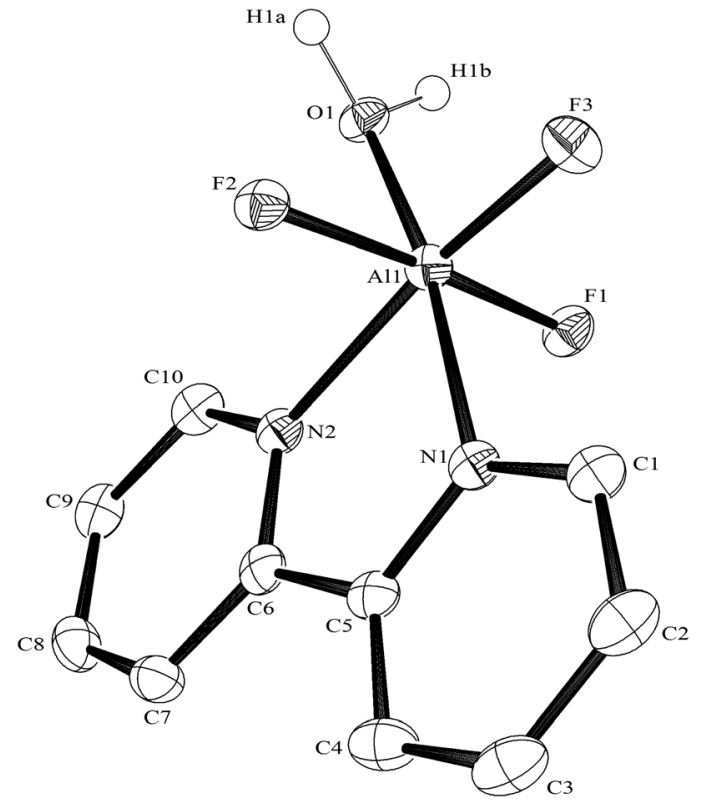

Fig. 9 The structure of the Al complex in $\left[\mathrm{AlF}_{3}(\right.$ bipy $\left.)\left(\mathrm{OH}_{2}\right)\right] \cdot 2 \mathrm{H}_{2} \mathrm{O}$ with ellipsoids drawn at the $50 \%$ probability level. Hydrogen atoms (except those on the coordinated water) are omitted for clarity. Selected bond lengths $(\AA)$ and angles $\left({ }^{\circ}\right)$ : Al1-F1 = 1.786(2), Al1-F2 = 1.781(2), Al1-F3 = 1.743(2), Al1-O1 $=1.900(2), A l 1-N 1=2.050(3), A l 1-N 2=2.072(3)$, $\mathrm{F} 2-\mathrm{Al1}-\mathrm{F} 3=93.35(11), \mathrm{F} 1-\mathrm{Al} 1-\mathrm{F} 3=92.22(11), \mathrm{F} 3-\mathrm{Al1}-\mathrm{O} 1=97.24(10)$, $\mathrm{F} 2-\mathrm{Al} 1-\mathrm{O} 1=88.30(11), \mathrm{F} 1-\mathrm{Al} 1-\mathrm{O} 1=89.16(11), \mathrm{F} 3-\mathrm{Al1}-\mathrm{N} 1=92.19(10)$, $\mathrm{F} 2-\mathrm{Al} 1-\mathrm{N} 1=90.08(10), \mathrm{F} 1-\mathrm{Al} 1-\mathrm{N} 1=91.55(10), \mathrm{F} 2-\mathrm{Al} 1-\mathrm{N} 2=86.50(11)$, $\mathrm{F} 1-\mathrm{Al} 1-\mathrm{N} 2$ = 88.32(11), O1-Al1-N2 = 92.39(11), N1-Al1-N2 = 78.18(11).

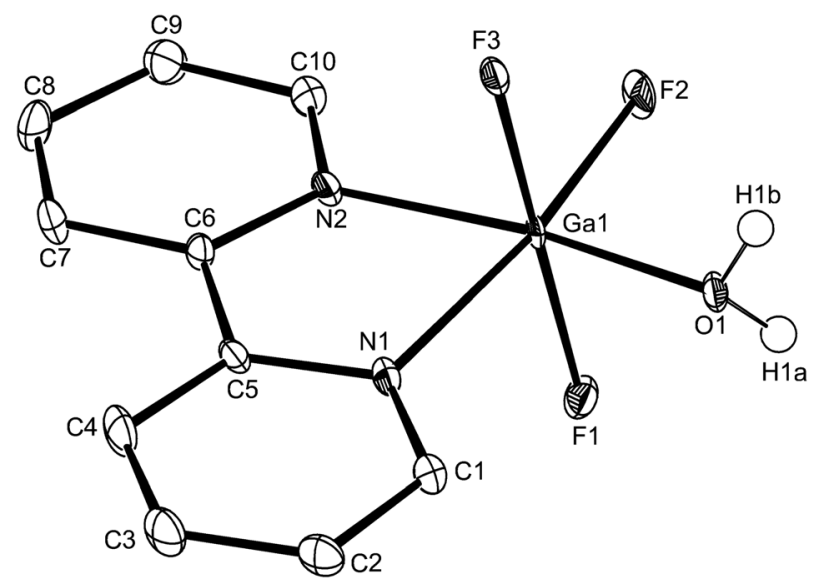

Fig. 10 The structure of $\mathrm{Ga}$ species in $\left[\mathrm{GaF}_{3}(\right.$ bipy $\left.)\left(\mathrm{OH}_{2}\right)\right] \cdot 2 \mathrm{H}_{2} \mathrm{O}$ with ellipsoids drawn at the $50 \%$ probability level. Hydrogen atoms (except those on the coordinated water) are omitted for clarity. Selected bond lengths $(\AA \AA)$ and angles $\left({ }^{\circ}\right)$ : Ga1-F1 = 1.8892(15), Ga1-F2 = 1.8442(17), $\mathrm{Ga1}-\mathrm{F} 3=1.8857(14), \mathrm{Ga} 1-\mathrm{O} 1=1.962(2), \mathrm{Ga} 1-\mathrm{N} 1=2.093(2), \mathrm{Ga} 1-\mathrm{N} 2=$ 2.064(3), F2-Ga1-F3 = 92.41(7), F1-Ga1-F2 = 90.74(7), F1-Ga1-F3 = $175.68(7), \mathrm{F} 2-\mathrm{Ga} 1-\mathrm{O} 1=96.70(8), \mathrm{F} 3-\mathrm{Ga} 1-\mathrm{O} 1=87.76(7), \mathrm{F} 1-\mathrm{Ga} 1-\mathrm{O} 1=$ 88.94(7), F2-Ga1-N2 = 92.28(9), F3-Ga1-N2 = 90.64(8), F1-Ga1-N2 = 92.19(8), F3-Ga1-N1 = 87.52(7), F1-Ga1-N1 = 89.86(7), O1-Ga1-N1 = 92.67(8), N2-Ga1-N1 = 78.35(9). 


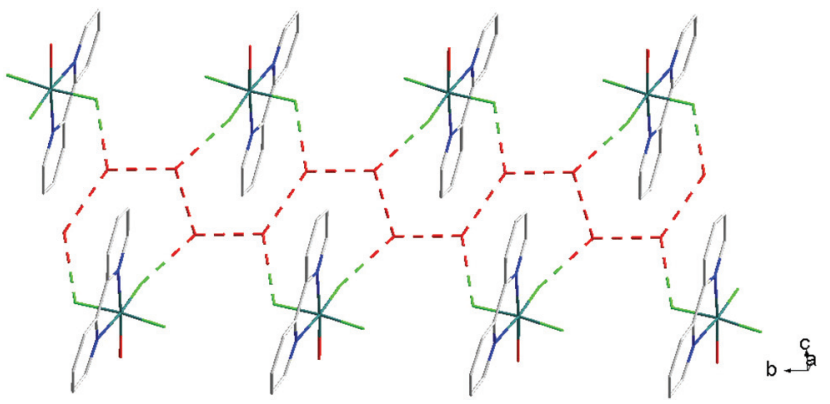

Fig. 11 View showing the $\mathrm{H}$-bonding network (red) involving the $\mathrm{F}^{-}$ligands and lattice $\mathrm{H}_{2} \mathrm{O}$ present in the structure of $\left[\mathrm{AlF}_{3}(\mathrm{bipy})-\right.$ $\left.\left(\mathrm{OH}_{2}\right)\right] \cdot 2 \mathrm{H}_{2} \mathrm{O}$.

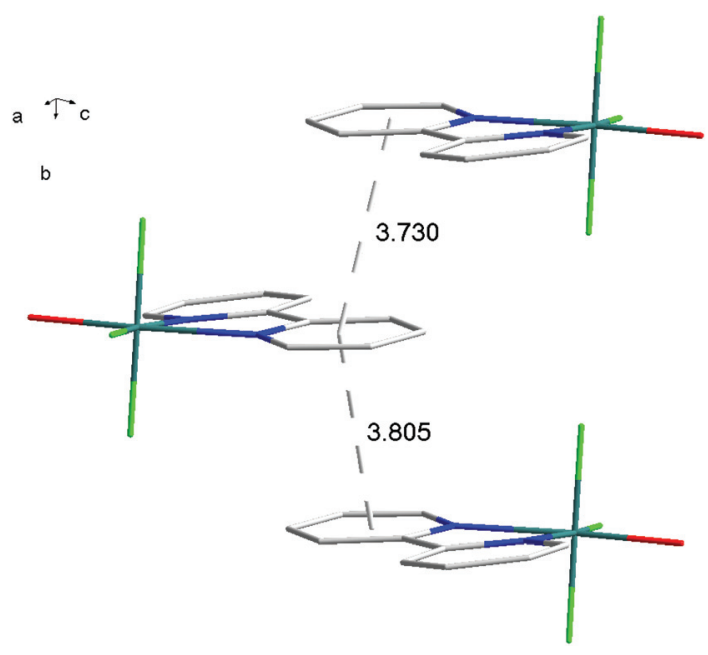

Fig. 12 View showing the $\pi$-stacking (lattice $\mathrm{H}_{2} \mathrm{O}$ omitted for clarity) present in the structure of $\left[\mathrm{AlF}_{3}(\right.$ bipy $\left.)\left(\mathrm{OH}_{2}\right)\right] \cdot 2 \mathrm{H}_{2} \mathrm{O}$.

Overall, the structural data reinforce earlier conclusions that trends in the bond lengths in comparable $\mathrm{Al}$ and $\mathrm{Ga}$ complexes often differ from those predicted on the basis of simple Lewis acidity in the gas phase $;^{22,27-29}$ other factors, including the presence or absence of lattice solvent and hydrogen bonding also need to be considered. ${ }^{13,19,21,22}$ The effects are less noticeable at indium, where corresponding bonds are typically $\sim 0.2 \AA$ longer than for Ga, reflecting the increased radius of the metal centre.

Using the same hydrothermal route with 1,10-phenanthroline in place of 2,2'-bipyridyl, gave $\left[\mathrm{MF}_{3}(\mathrm{phen})\left(\mathrm{OH}_{2}\right)\right]$, notably without lattice water (in contrast to the terpy and bipy complexes above). The structure of crystals of $\left[\mathrm{GaF}_{3}(\mathrm{phen})\left(\mathrm{OH}_{2}\right)\right]$ is shown in Fig. 14, and the $\left[\operatorname{InF}_{3}\right.$ (phen) $\left.\left(\mathrm{OH}_{2}\right)\right]$ is isomorphous. ${ }^{13}$ Corresponding bond lengths are again $\sim 0.15-0.20 \AA$ A longer for the indium complex. The $\left[\mathrm{GaF}_{3}(\mathrm{phen})\left(\mathrm{OH}_{2}\right)\right]$ also shows $\pi$-stacking of the aromatic rings (Fig. S3 $\$$ ) and intermolecular $\mathrm{F} \cdot \mathrm{H}$ H hydrogen bonding, in this case involving the coordinated water molecules (Fig. 15).

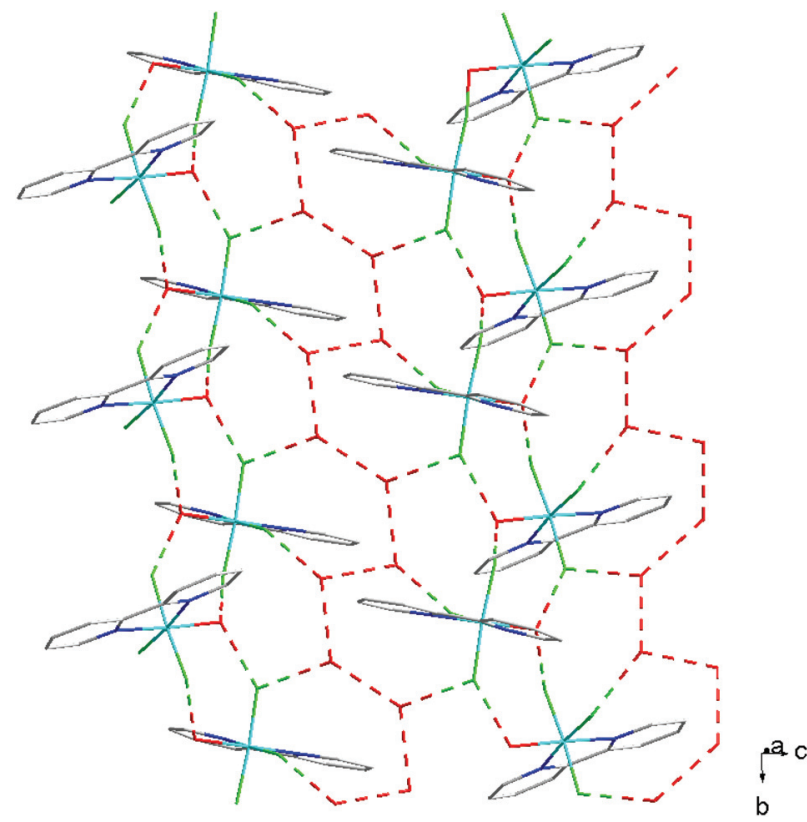

Fig. 13 View showing the $\mathrm{H}$-bonding network (red) present in the structure of $\left[\mathrm{GaF}_{3}(\right.$ bipy $\left.)\left(\mathrm{OH}_{2}\right)\right] \cdot 2 \mathrm{H}_{2} \mathrm{O}$.

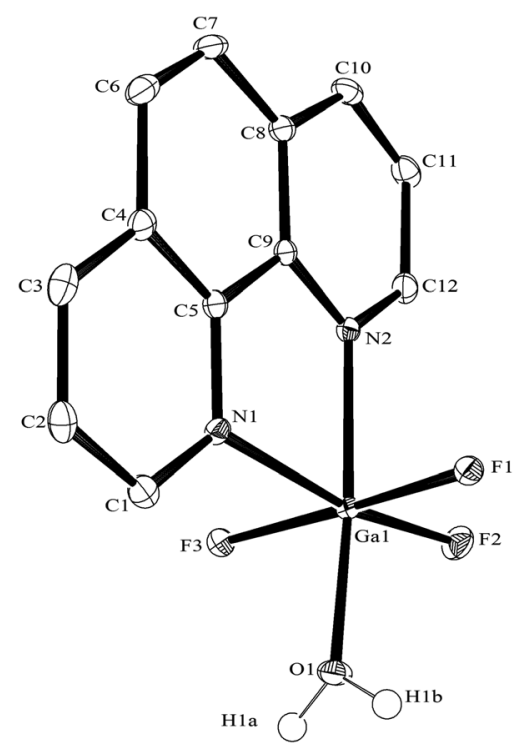

Fig. 14 The structure of $\left[\mathrm{GaF}_{3}\right.$ (phen) $\left.\left(\mathrm{OH}_{2}\right)\right]$ with ellipsoids drawn at the $50 \%$ probability level. Hydrogen atoms (except those on the coordinated water) are omitted for clarity. Selected bond lengths $(\AA)$ and angles $\left({ }^{\circ}\right)$ : Ga1-F1 = 1.8990(11), Ga1-F2 = 1.8242(12), Ga1-F3 = 1.8790(11), Ga1-N1 $=2.1250(16), \mathrm{Ga} 1-\mathrm{N} 2=2.0897(16), \mathrm{Ga} 1-\mathrm{O} 1=1.9594(14), \mathrm{F} 2-\mathrm{Ga} 1-\mathrm{F} 3=$ 95.13(5), F1-Ga1-F2 = 93.69(5), F2-Ga1-O1 = 98.11(6), F3-Ga1-O1 = 89.79(5), F1-Ga1-O1 = 86.92(5), F2-Ga1-N2 = 92.02(6), F3-Ga1-N2 = 93.11(5), F1-Ga1-N2 = 88.65(5), F3-Ga1-N1 = 87.18(6), F1-Ga1-N1 = 84.47(6), O1-Ga1-N1 = 91.54(6), N2-Ga1-N1 = 78.20(6).

The IR spectra of the $\left[\mathrm{MF}_{3}(\right.$ phen $\left.)\left(\mathrm{OH}_{2}\right)\right]$ complexes show quite weak features due to $\nu(\mathrm{OH})$ and $\delta(\mathrm{HOH})$, whereas in the hydrated $\left[\mathrm{MF}_{3}(\right.$ bipy $\left.)\left(\mathrm{OH}_{2}\right)\right] \cdot 2 \mathrm{H}_{2} \mathrm{O}$ the corresponding features 


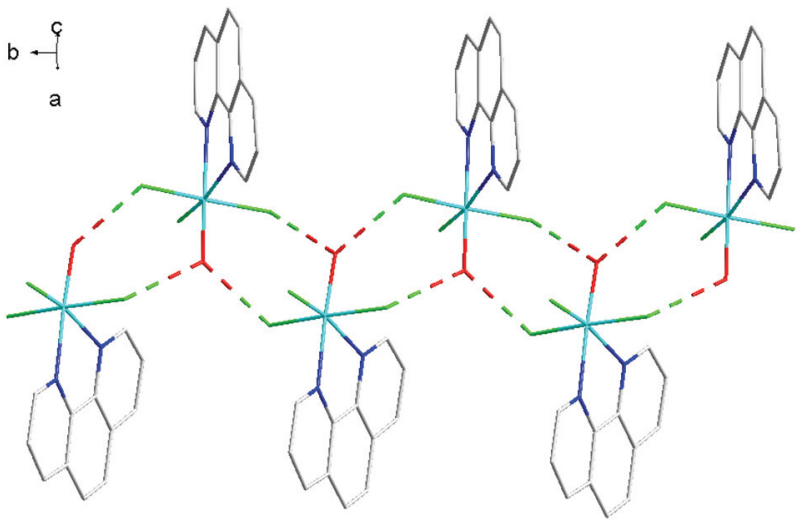

Fig. 15 View showing the $\mathrm{H}$-bonding network (red) present in the structure of $\left[\mathrm{GaF}_{3}(\right.$ phen $\left.)\left(\mathrm{OH}_{2}\right)\right]$.

are much stronger and show several overlapping bands. The $\nu(\mathrm{MF})$ fall in energy as expected $\mathrm{Al}>\mathrm{Ga}>\mathrm{In}$, but for the corresponding $\mathrm{Al}$ and $\mathrm{Ga}$ complexes (less clearly in the In complexes), those in $\left[\mathrm{MF}_{3}\left(\right.\right.$ bipy) $\left.\left(\mathrm{OH}_{2}\right)\right] \cdot 2 \mathrm{H}_{2} \mathrm{O}$ occur at lower frequency than in $\left[\mathrm{MF}_{3}\right.$ (phen) $\left.\left(\mathrm{OH}_{2}\right)\right]$, which is probably due to the extensive hydrogen bonding in the former.

The multinuclear NMR data show interesting trends as the metal is changed. The complexes were insoluble or very poorly soluble in chlorocarbons and $\mathrm{CD}_{3} \mathrm{CN}$, and therefore spectra were obtained from freshly prepared solutions in $\mathrm{CD}_{3} \mathrm{OD}$. The solutions decompose slowly forming $\mathrm{F}^{-}$or $\mathrm{HF}_{2}{ }^{-}$, qualitatively the indium complexes seem less stable in solution than those of the lighter metals. At $183 \mathrm{~K}$, all six complexes show two resonances with relative intensities $1: 2$ in the ${ }^{19} \mathrm{~F}\left\{{ }^{1} \mathrm{H}\right\}$ NMR spectra, consistent with the solid state structures. On warming, the fluorine resonances of the indium complexes are lost, and those of the gallium complexes and of $\left[\mathrm{AlF}_{3}\right.$ (bipy)$\left.\left(\mathrm{OH}_{2}\right)\right]$ broaden and then coalesce; only the ${ }^{19} \mathrm{~F}\left\{{ }^{1} \mathrm{H}\right\}$ NMR spectrum of $\left[\mathrm{AlF}_{3}\right.$ (phen) $\left.\left(\mathrm{OH}_{2}\right)\right]$ shows two fluorine resonances at room temperature. The ${ }^{1} \mathrm{H}$ NMR spectra of the aluminium complexes show inequivalent pyridyl rings, as expected from the static structures, but those of the gallium and indium show broad resonances even at low temperatures. The temperature dependence of the spectra is consistent with reversible ligand dissociation, possibly of the water, to give a fluxional five-coordinate intermediate, the dissociation increasing with decreased Lewis acid strength $\mathrm{Al}>\mathrm{Ga}>\mathrm{In}$.

\section{Reactions of $\mathrm{MF}_{3} \cdot 3 \mathrm{H}_{2} \mathrm{O}$ with PMDTA}

PMDTA, $\mathrm{Me}_{2} \mathrm{~N}\left(\mathrm{CH}_{2}\right)_{2} \mathrm{NMe}\left(\mathrm{CH}_{2}\right)_{2} \mathrm{NMe}_{2}$, is a flexible, aliphatic acyclic triamine analogue of $\mathrm{Me}_{3}$-tacn and terpy, and its reactions with the hydrated Group 13 fluorides were explored to provide a third series of complexes for comparison. In contrast to the reactions with the other two $\mathrm{N}_{3}$-donor ligands, the hydrothermal route using PMDTA resulted in cleavage of the triamine and the formation of the 1,1,4-trimethylpiperazinium cation, $\left[\subset \mathrm{Me}_{2} \mathrm{~N}\left(\mathrm{CH}_{2}\right)_{2} \mathrm{NMe}\left(\mathrm{CH}_{2}\right)_{2}\right]^{+}$. After removing all volatiles from the reaction mixtures and washing the residue with

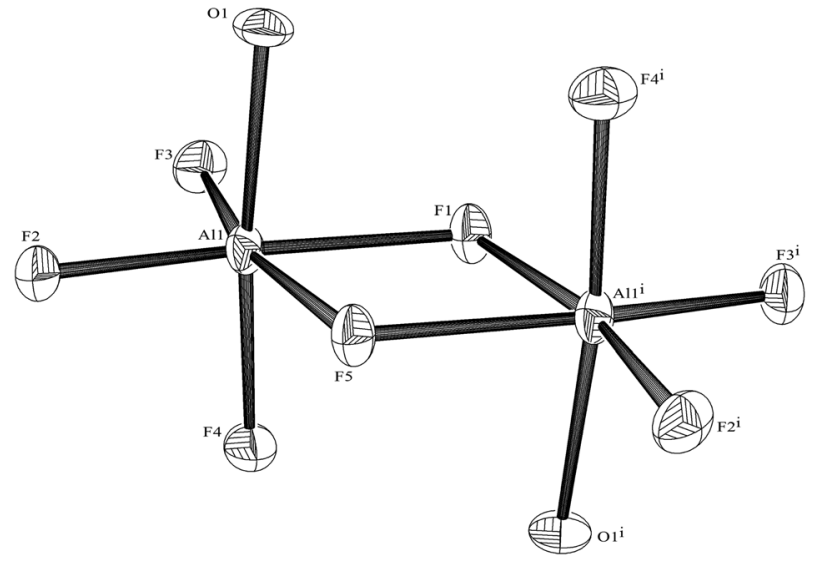

Fig. 16 The structure of the $\left[\mathrm{Al}_{2} \mathrm{~F}_{8}\left(\mathrm{OH}_{2}\right)_{2}\right]^{2-}$ anion with ellipsoids drawn at the $50 \%$ probability level and with $(\mathrm{O}-\mathrm{H})$ hydrogens omitted. Selected bond lengths $(\AA)$ and angles $\left({ }^{\circ}\right)$ : Al1-F2 = 1.748(2), Al1- F3 = 1.749(2), $\mathrm{Al} 1-\mathrm{F} 4=1.778(2), \mathrm{Al}-\mathrm{O} 1=1.895(2), \mathrm{Al} 1-\mathrm{F} 5=1.8968(19), \mathrm{Al} 1-\mathrm{F} 1=$ 1.9048(19), F2-Al1-F3 = 99.65(11), F2-Al1-F4 = 93.28(10), F3-Al1-F4 = 93.83(10), F2-Al1-O1 = 90.62(10), F3-Al1-O1 = 90.65(10), F2-Al1-F5 = 92.34(10), F4-Al1-F5 = 88.95(8), O1-Al1-F5 = 85.68(8), F3-Al1-F1 = 91.94(10), F4-Al1-F1 = 88.61(8), O1-Al1-F1 = 86.52(8), F5-Al1-F1 =

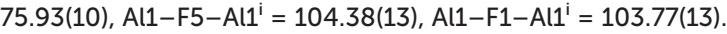

MeCN, the ${ }^{1} \mathrm{H}$ and ${ }^{13} \mathrm{C}\left\{{ }^{1} \mathrm{H}\right\}$ NMR spectra show the cyclic cation to be the only organic species in the bulk products. For the aluminium reaction, crystals were obtained, showing the solid contained $\left[\subset \mathrm{Ce}_{2} \mathrm{~N}\left(\mathrm{CH}_{2}\right)_{2} \mathrm{NMe}\left(\mathrm{CH}_{2}\right)_{2}\right]_{2}\left[\mathrm{Al}_{2} \mathrm{~F}_{8}\left(\mathrm{OH}_{2}\right)_{2}\right] \cdot 2 \mathrm{H}_{2} \mathrm{O}$. The anion (Fig. 16) has been obtained previously as the $\left[\mathrm{NMe}_{4}\right]^{+}$ salt, ${ }^{32}$ and breaks up in solution to form $\left[\mathrm{AlF}_{4}\right]^{-}$, which was identified by a combination of ${ }^{27} \mathrm{Al}(\delta=48.7(\mathrm{~s}))$ and ${ }^{19} \mathrm{~F}\left\{{ }^{1} \mathrm{H}\right\}$ NMR data $\left(\delta=-194.6,6\right.$ lines, $\left.{ }^{1} J_{\mathrm{AlF}}=38 \mathrm{~Hz}\right) .{ }^{32}$

In the cases of the gallium and indium reactions, a few crystals of the same cation were obtained as the chloride salt, from traces of chloride in the reaction. The structure of $\left[\subset \mathrm{Me}_{2} \mathrm{~N}\right.$ $\left.\left(\mathrm{CH}_{2}\right)_{2} \mathrm{NMe}\left(\mathrm{CH}_{2}\right)_{2}\right] \mathrm{Cl}$ has been reported previously ${ }^{31}$ and the crystals obtained in this study were identical, and hence are not discussed further (see ESI + ). The data on the bulk product from the gallium reaction fitted the constitution $\left[\subset \mathrm{Me}_{2} \mathrm{~N}\right.$ $\left.\left(\mathrm{CH}_{2}\right)_{2} \mathrm{NMe}\left(\mathrm{CH}_{2}\right)_{2}\right]_{2}\left[\mathrm{Ga}_{2} \mathrm{~F}_{8}\left(\mathrm{OH}_{2}\right)_{2}\right] \cdot 2 \mathrm{H}_{2} \mathrm{O}$, analogous to the aluminium complex, although in the absence of X-ray structural data, the anion present cannot be confirmed. The relative instability of the fluoro-metallate anions in solution and the sensitivity of the ${ }^{19} \mathrm{~F}$ chemical shifts to solvent, ${ }^{30,32}$ make identification of the anions uncertain without structural data. The reactions of the $\mathrm{MF}_{3} \cdot 3 \mathrm{H}_{2} \mathrm{O}$ with PMDTA were also attempted in refluxing methanol solution, since it was reasoned that the milder conditions (compared to the hydrothermal preparations) might have prevented cleavage of the PMDTA. No reaction occurred in the case of indium fluoride, whilst with $\mathrm{AlF}_{3} \cdot 3 \mathrm{H}_{2} \mathrm{O},{ }^{1} \mathrm{H}$ and ${ }^{19} \mathrm{~F}\left\{{ }^{1} \mathrm{H}\right\}$ NMR spectra of the crude product showed protonated PMDTA and $\left[\mathrm{AlF}_{4}\right]^{-}$as the only significant species. The contrast between the instability of PMDTA and the robust $\mathrm{Me}_{3}$-tacn which has similar groups in these Group 13 fluoride reactions may be due to the ring structure of the latter preventing close approach of an amine 
function polarised by coordination to the metal, to the next $\mathrm{CH}_{2} \mathrm{NMe}$ unit, which is presumably the first stage in $\mathrm{C}-\mathrm{N}$ bond fission and formation of the small ring.

\section{Conclusions}

Hydrothermal synthesis using $\mathrm{MF}_{3} \cdot 3 \mathrm{H}_{2} \mathrm{O}$ as the metal source has been shown to be a convenient method to form a range of complexes of the otherwise rather intractable Group 13 trifluorides with nitrogen heterocycles in high yield. X-Ray crystallographic studies show all of the new complexes contain a mer arrangement of fluorides, contrasting with the fac geometry present in the triaza-macrocyclic complexes ${ }^{15}$ reported previously. Extensive $\mathrm{H}$-bonding and $\pi$-stacking networks are present in the complexes of all three imines with the three metal ions, although the details differ. These studies significantly extend the known coordination chemistry of the Group 13 trifluorides. The relatively high stability of the trifluoride complexes contrasts with the moisture sensitivity of complexes of the Group 13 elements with heavier halides. However, this work has also shown that unlike $\left[\mathrm{GaF}_{3}\left(\mathrm{BzMe}_{2}\right.\right.$-tacn $\left.)\right] \cdot 4 \mathrm{H}_{2} \mathrm{O}$, which is stable towards alkali metal or ammonium salts in aqueous MeCN, forming heterometallic (or mixed galliumammonium) complexes, competitive ions such as $\left[\mathrm{NH}_{4}\right]^{+}$ and $\left[\mathrm{PF}_{6}\right]^{-},\left[\mathrm{GaF}_{3}(\right.$ terpy) $] \cdot 3 \mathrm{H}_{2} \mathrm{O}$ reacts, with loss of a fluoride ligand, to form the dinuclear product, $\left[\{\mathrm{Ga}(\text { terpy }) \mathrm{F}\}_{2}(\mu-\mathrm{F})_{2}\right]$ $\left[\mathrm{PF}_{6}\right]_{2} \cdot 4 \mathrm{H}_{2} \mathrm{O}$.

Future work will aim to establish whether the hydrothermal approach is also suitable for oxygen donor ligands and whether soft donor ligands such as thioethers or phosphines can form complexes with the Group 13 fluorides.

\section{Acknowledgements}

We thank EPSRC and GE Healthcare for support (EP/I501673/1, EP/L505651/1 and EP/1033394/1).

\section{References}

1 (a) S. L. Benjamin, W. Levason and G. Reid, Chem. Soc. Rev., 2013, 42, 1460; (b) Chemistry of aluminium, gallium, indium and thallium, ed. A. J. Downs, Blackie, London, 1st edn, 1993.

2 (a) E. Kemnitz, U. Gross, St. Ruediger, G. Scholz, D. Heideman, S. I. Troyanov, I. V. Morosov and M.-H. Lemee-Cailleau, Solid State Sci., 2006, 8, 1443; (b) P. Bukovec and V. Kaucic, Inorg. Nucl. Chem. Lett., 1978, 14, 79.

3 S. Petricek, A. Demsar, P. Bukovec, L. Golic and J. V. Brencic, Acta Chim. Slov., 1997, 44, 317.

4 F. N. Penkert, T. Weyhermuller and K. Wieghardt, Chem. Commun., 1998, 557.

5 D. R. Ketchum, G. L. Schimek, W. T. Pennington and J. W. Kolis, Inorg. Chim. Acta, 1999, 294, 200.
6 (a) M. Roos and G. Meyer, Z. Anorg. Allg. Chem., 1999, 625, 1129. M. Roos and G. Meyer, Z. Anorg. Allg. Chem., 1999, 625, 1843.

7 S. A. Polyshchuk, S. P. Kozerenko and Y. V. Gagarinsky, J. Less-Common Met., 1972, 27, 45.

8 S. P. Kozerenko, S. A. Polyshchuk and N. I. Sigula, Russ. J. Inorg. Chem., 1972, 17, 954.

9 M. Roos, J. Wittrock, G. Meyer, S. Fritz and J. Straehle, Z. Anorg. Allg. Chem., 2000, 626, 1179.

10 S. P. Petrosyants and E. R. Buslaeva, Russ. J. Inorg. Chem., 1989, 34, 932.

11 S. A. Polyshchuk and G. F. Krysenko, Koord. Khim., 1977, 3, 816.

12 M. A. Malyarik, S. P. Petrosyants, A. B. Ilyukhin and Yu. A. Buslaev, Russ. J. Inorg. Chem., 1991, 36, 2816.

13 (a) A. B. Ilyukhin and M. A. Malyarik, Koord. Khim., 1999, 44, 1511; (b) A. B. Ilyukhin and M. A. Malyarik, Krystallographiya, 1994, 39, 439.

14 S. P. Petrosyants and A. B. Ilyukhin, Russ. J. Inorg. Chem., 2010, 55, 30 .

15 R. Bhalla, C. Darby, W. Levason, S. K. Luthra, G. McRobbie, G. Reid, G. W. Sanderson and W. Zhang, Chem. Sci., 2014, 5,381 .

16 (a) A. Dimitrov, D. Heidemann, K. I. Khallow and E. Kemnitz, Inorg. Chem., 2012, 51, 11612; (b) A. Dimitrov, D. Heidemann and E. Kemnitz, Inorg. Chem., 2006, 45, 10807.

17 (a) P. Laverman, W. McBride, R. Sharkey, A. Eek, L. Joosten, W. Oyen, D. Goldenberg and O. Boerman, J. Nucl. Med., 2010, 51, 454; (b) W. McBride, C. D'Souza, R. Sharkey, H. Karacay, E. Rossi, C. Chang and D. Goldenberg, Bioconjugate Chem., 2010, 21, 1331; (c) W. McBride, R. Sharkey, H. Karacay, C. D’Souza, E. Rosso, P. Laverman, C. Chang, O. Boerman and D. Goldenberg, J. Nucl. Med. Chem., 2009, 50, 991; (d) W. McBride, C. D’Souza, H. Karacay, R. Sharkey and D. Goldenberg, Bioconjugate Chem., 2012, 23, 538; (e) D. Shetty, S. Y. Choi, J. M. Jeong, J. Y. Lee, L. Hoigebazar, Y.-S. Lee, D. S. Lee, J. K. Chung, M. C. Lee and Y. K. Chung, Chem. Commun., 2011, 47, 9732; (f) R. Bhalla, W. Levason, S. K. Luthra, G. McRobbie, G. Sanderson and G. Reid, Chem. - Eur. J., 2015, 21, 4688.

18 G. M. Sheldrick, Acta Crystallogr., Sect. A: Fundam. Crystallogr., 2008, 64, 112.

19 I. V. Kazakov, M. Bodensteiner and A. Y. Timoshkin, Acta Crystallogr., Sect. C: Cryst. Struct. Commun., 2014, 70, 312.

20 (a) M. J. Taylor and P. J. Brothers, in The chemistry of aluminium, gallium, indium and thallium, ed. and A. J. Downs, Blackie, London, 1993, p. 111; (b) B. Cordero, V. Gomez, A. E. Platero-Prats, M. Reves, J. Echeverria, E. Cremades, F. Barragan and S. Alvarez, Dalton Trans., 2008, 2832.

21 (a) J. Burt, W. Levason, M. E. Light and G. Reid, Dalton Trans., 2014, 43, 14600; (b) K. George, M. Jura, W. Levason, M. E. Light and G. Reid, Dalton Trans., 2014, 43, 3637.

22 A. Y. Timoshkin, M. Bodensteiner, T. N. Sevastianova, A. S. Lisovenko, E. I. Davydova, M. Scheer, C. Grassi and A. V. Butlak, Inorg. Chem., 2012, 51, 11602.

$23{ }^{27} \mathrm{Al}[\mathrm{I}=5 / 2,100 \%$ abundance, $\Xi=26.08 \mathrm{MHz}, Q=0.149 \times$ $\left.10^{-28} \mathrm{~m}^{2}, R_{\mathrm{C}}=1170\right],{ }^{69} \mathrm{Ga}[I=3 / 2,60.4 \%$ abundance, $\Xi=$ 
24.07 MHz, $\left.Q=0.178 \times 10^{-28} \mathrm{~m}^{2}, R_{\mathrm{C}}=237\right]{ }^{71} \mathrm{Ga}[I=3 / 2$, $39.6 \%$ abundance, $\Xi=30.58 \mathrm{MHz}, \mathrm{Q}=0.112 \times 10^{-28} \mathrm{~m}^{2}, R_{\mathrm{C}}=$ 322 $]{ }^{115} \mathrm{In}[I=9 / 2,95.7 \%$ abundance, $\Xi=22.04 \mathrm{MHz}, Q=$ $\left.1.16 \times 10^{-28} \mathrm{~m}^{2}, R_{\mathrm{C}}=1920\right]$.

24 J. Mason, Multinuclear NMR, Plenum, New York, 1987.

25 R. Bhalla, W. Levason, S. J. Luthra, G. McRobbie, G. Reid, G. Sanderson and W. Zhang, Chem. Commun., 2014, 50, 12673.

26 K. S. Pedersen, G. Lorusso, J. J. Morales, T. Weyhermuller, S. Piligkos, S. K. Singh, D. Larsen, M. Schau-Magnussen, G. Rajaraman, M. Evanelisti and J. Bendix, Angew. Chem., Int. Ed., 2014, 53, 2394.
27 F. Bessac and G. Frenking, Inorg. Chem., 2006, 45, 6956.

28 J. Burt, W. Levason and G. Reid, Coord. Chem. Rev., 2014, 260, 65, and refs therein.

29 A. V. Timoshkin, A. V. Suvorov, H. F. Bettinger and H. F. Schaefer III, J. Am. Chem. Soc., 1999, 121, 5687.

30 (a) N. Herron, D. L. Thorn, R. L. Harlow and F. Davidson, J. Am. Chem. Soc., 1993, 115, 3028; (b) N. Herron, R. L. Harlow and D. L. Thorn, Inorg. Chem., 1993, 32, 2985.

31 W. Massa and K. Dehnicke, Z. Anorg. Allg. Chem., 2009, 635, 287.

32 S. P. Petrosyants and A. B. Ilyukhin, Russ. J. Inorg. Chem., 2011, 56, 1250. 